\title{
Online Optimization with Predictions and
}

\section{Switching Costs: Fast Algorithms and the Fundamental Limit}

\author{
Yingying Li, Guannan $\mathrm{Qu}$, and $\mathrm{Na} \mathrm{Li}$
}

\begin{abstract}
This paper studies an online optimization problem with a finite prediction window of cost functions and additional switching costs on decisions. We propose two gradient-based online algorithms: Receding Horizon Gradient Descent (RHGD), and Receding Horizon Accelerated Gradient (RHAG). Both algorithms only require a finite number of projected gradient evaluations at each stage. We provide upper bounds on the dynamic regrets of the proposed algorithms and show that the regret upper bounds decay exponentially with the length of the prediction window. Moreover, we study the fundamental lower bound on the dynamic regret for a broad class of deterministic online algorithms. The lower bound is close to RHAG's regret upper bound, indicating that our gradient-based RHAG is a near-optimal online algorithm. Finally, we conduct numerical experiments to complement our theoretical analysis.
\end{abstract}

\section{INTRODUCTION}

In many applications of sequential decision-making problems, e.g. data center scheduling [1], smart grids [2], multi-task machine learning [3], autonomous driving [4], etc., the system is subject to a time-varying environment and only the near future can be predicted with high accuracy; meanwhile, abrupt and large changes in the decisions are undesirable. Inspired by this, in this paper, we consider an online convex optimization problem with a short-term prediction of the cost functions and additional switching costs on the decisions. In particular, at each stage $t$, an agent receives the cost functions for the next $W$ stages, i.e. $f_{t}, \ldots, f_{t+W-1}$, and then makes a decision $x_{t}$. The agent suffers the stage cost $f_{t}\left(x_{t}\right)$ and also a switching cost $\frac{\beta}{2}\left\|x_{t}-x_{t-1}\right\|^{2}$ that penalizes the change in the decision at stage $t$, where $\beta \geq 0$ is a weight parameter.

This problem is a variant of the classical online convex optimization (OCO) that does not consider predictions or switching costs [5]. This variant has attracted a lot of attention from the OCO community in recent years. Many algorithms have been proposed and their optimality/regret guarantees have been analyzed, e.g. AFHC [1], CHC [6]. In addition, though not specially designed for this problem, a classical control algorithm, model predictive

The work was supported by NSF 1608509, NSF CAREER 1553407, AFOSR YIP, and ARPA-E through the NODES program. Y. Li, G. Qu and N. Li are with John A. Paulson School of Engineering and Applied Sciences, Harvard University, 33 Oxford Street, Cambridge, MA 02138, USA (email: yingyingli@g.harvard.edu, gqu@g.harvard.edu, nali@seas.harvard.edu). A preliminary version of this paper appears in the 2018 American Control Conference. The current version contains a new algorithm with better performance and refined fundamental limit results. 
control (MPC) [7], can be naturally applied here because MPC considers the receding horizon optimization for the next $W$ stages and the information of the next $W$ stages is available in our setting. However, most algorithms in literature, e.g. AFHC, $\mathrm{CHC}, \mathrm{MPC}$, require to solve $W$-stage optimization at each stage, which can be timeconsuming for large-scale problems. Moreover, despite the attempts on relieving the computational burden of MPC, e.g. explicit MPC [8], inexact MPC [9], suboptimal MPC [10], [11], prediction-correction methods [12], real-time iteration schemes [13], etc., most existing methods either require special structures of the cost functions or lack optimality/regret guarantees for the online problems with time-varying costs.

Further, questions regarding the fundamental limit of this problem are relatively under-explored in the literature. In this paper, we are mostly interested in the following question.

$Q:$ what is achievable and what is not by online algorithms that have a finite window of the cost predictions?

Contributions: This paper considers strongly convex and smooth cost functions and studies the performance by dynamic regret, which is the online algorithm's cost minus the optimal cost in hindsight [14]. Our results are summarized below.

Firstly, we design gradient-based online algorithms, RHGD and RHAG. Our algorithms adopt classical OCO algorithms (without predictions), e.g. online gradient descent (OGD) [5], as an initialization oracle, and then performs gradient updates on the initialization based on the cost predictions. With OGD initialization, our algorithms only require the calculation of $W+1$ gradients. Moreover, we show theoretically that our algorithms reduce the regret of the initialization oracle, which can be any classical OCO algorithm, exponentially with the prediction window's length $W$. We also provide some stability guarantees of our algorithms under certain conditions.

In addition, we analyze the fundamental lower bounds on the dynamic regrets of any online algorithms, which is based on the worst-case analysis and imposes no computational constraints on the admissible algorithms. Surprisingly, the regret upper bound of RHAG is close to the fundamental lower bound, indicating that, at least in the worst cases, our gradient-based RHAG algorithm is near-optimal even when compared with more computationally intensive algorithms.

Finally, we conduct numerical experiments to complement our theoretical analysis by comparing our algorithms with MPC and discuss the implications of the numerical results.

Related work: We provide a brief literature review below.

(Economic) model predictive control. The problem considered in this paper is related with the (economic) model predictive control ((E)MPC) with time-varying costs [15]-[21], where EMPC is a variant of MPC that focuses on minimizing the economic costs (see e.g. [17]). In addition, our dynamic regret analysis is related with the optimality performance analysis of (E)MPC that studies how MPC's cost deviates from the optimal one [16]-[19]. However, the optimality performance of the fast (E)MPC schemes (see e.g. [8]-[13]) is relatively under-explored, especially for the time-varying cases.

It is worth mentioning that MPC can be applied to much more general problems, e.g. dynamical control problems, systems with constraints, distributed systems, etc. (see [7] for more details), while this paper only considers OCO with switching costs. Nevertheless, this paper may lay foundation for more general problems, e.g. the online optimal control with a linear time-invariant system as in [22]. 
Online convex optimization. For classical OCO, we refer the reader to [5]. The OCO with switching costs has been studied in [1], [6], [23]. The OCO with predictions has been studied for the case without switching costs where one-stage inaccurate predictions are considered (see e.g. [24]), and for the case with switching costs and multi-stage predictions which are either accurate [1] or inaccurate [6]. This paper is mostly related with the work on the $W$-stage accurate prediction [1].

Various performance metrics are considered in OCO, e.g. static regret [5], dynamic regret [14], [25], competitive ratio [26], etc. The static regret refers to the difference between the online algorithm's cost and the cost generated by an optimal static decision in hindsight. There are algorithms with sublinear $o(T)$ static regret bounds [5]. However, with large fluctuations in the environment, it is also reasonable to compare with the cost of the possibly timevarying optimal decisions in hindsight, as considered in the dynamic regret and the competitive ratio. This paper will focus on the dynamic regret and leave the competitive ratio analysis as the future work. Notice that it is usually impossible to achieve a sublinear $o(T)$ dynamic regret bound for all time-varying environments and most dynamic regret bounds in literature depend on the variation of the environment [14], [25].

Time-varying optimization (TVO). It considers $\min _{x} f(x ; t)$ for each $t$. For theoretical purposes, most papers on TVO assume that the cost function $f(x ; t)$ does not change dramatically with time $t$, e.g. $f(x ; t)$ has certain smoothness properties with respect to $t$ [20], [27], [28], which are not assumed in this paper. It is also worth mentioning the prediction-correction method [27], which computes the predictions of the future costs based on the smoothness of $f(x ; t)$ with $t$; while the predictions in this paper are not computed by our algorithms but are given by some outside sources. It is our future work to consider designing algorithms to generate predictions.

Notations: $\|\cdot\|$ denotes the $l_{2}$ norm. $\Pi_{\mathbb{X}}(x)$ denotes the projection of $x$ onto set $\mathbb{X}$. For function $f(x, y)$ on $(x, y) \in \mathbb{R}^{m+n}$, let $\nabla f(x, y) \in \mathbb{R}^{m+n}$ be the gradient and $\frac{\partial f}{\partial x}(x, y) \in \mathbb{R}^{m}$ be the partial gradient with $x$. For integers $a, b, c$, we write $a \equiv b(\bmod c)$ if $a=b+k c$ for some integer $k$. $|\mathbb{J}|$ denotes the cardinality of the set J. $\mathbf{A}^{\top}$ denotes the matrix A's transpose. For $x>0$ in $\mathbb{R}$, we write $f(x)=O(g(x))(f(x)=\Omega(g(x)))$ if there exists a constant $M$ such that $|f(x)| \leq M g(x)(|f(x)| \geq M g(x))$ for $x \geq M$; and we write $f(x)=o(g(x))$ if $\lim _{x \rightarrow+\infty} f(x) / g(x)=0 . \mathbf{1}_{n} \in \mathbb{R}^{n}$ is an all-one vector. $\boldsymbol{I}_{n}$ is an identity matrix in $\mathbb{R}^{n \times n}$.

\section{Problem Formulation}

This paper considers an online convex optimization (OCO) problem in $T$ stages with stage cost function $f_{t}(\cdot)$ and quadratic switching cost $\frac{\beta}{2}\left\|x_{t}-x_{t-1}\right\|^{2}$ to penalize the changes in the decisions. Formally, we aim to solve

$$
\min _{x_{1}, \ldots, x_{T} \in \mathbb{X}} \mathrm{C}_{\mathrm{T}}(\boldsymbol{x})=\sum_{t=1}^{T}\left(f_{t}\left(x_{t}\right)+\frac{\beta}{2}\left\|x_{t}-x_{t-1}\right\|^{2}\right),
$$

where $\mathbb{X} \subseteq \mathbb{R}^{n}$ is a convex feasible set, $\boldsymbol{x}=\left(x_{1}, \ldots, x_{T}\right), x_{0} \in \mathbb{X}$ is given, $\beta \geq 0$ is a penalty parameter.

To solve (1), all cost functions $f_{1}, \ldots, f_{T}$ have to be known a priori, which is not practical in many applications [1]. Nevertheless, there are usually some predictions available, especially for the near future. This paper adopts a simple model to characterize the predictions: at each stage $t$, the decision maker receives the cost functions 
for the next $W$ stages $f_{t}, \ldots, f_{t+W-1},{ }^{1}$ but do not know the cost functions beyond the next $W$ stages, that is, $f_{t+W}, f_{t+W+1}, \ldots$ may be arbitrary or even adversarial. Though our prediction model is too optimistic in the near future but too pessimistic in the far future, our model captures a commonly observed property in applications, i.e. the short-range predictions are usually much more accurate than the long-range predictions. In addition, our model simplifies the theoretical analysis and helps generate insightful results that may lay foundation for future work on more realistic settings, e.g. noisy and/or partial predictions.

In summary, the online problem considered in this paper is outlined below. At each stage $t=1,2, \ldots, T$, an agent

- receives the predicted cost functions $f_{t}(\cdot), \ldots, f_{t+W-1}(\cdot)$

- computes a stage decision $x_{t}$ by history and predictions

- suffers the cost $f_{t}\left(x_{t}\right)+\frac{\beta}{2}\left\|x_{t}-x_{t-1}\right\|^{2}$.

The online information available at each stage $t$ contains the predicted cost functions as well as the history cost functions, i.e. $\left\{f_{1}, \ldots, f_{t+W-1}\right\}$. Our goal is to design an online algorithm $\mathscr{A}$ that computes the stage decision $x_{t}^{\mathscr{A}}$ by only using the online information at stage $t$ to minimize the total cost $\mathrm{C}_{\mathrm{T}}\left(\boldsymbol{x}^{\mathscr{A}}\right)$. We measure the algorithm performance by dynamic regret [14], which compares the online algorithm's cost with the optimal cost in hindsight:

$$
\operatorname{Reg}(\mathscr{A})=\mathrm{C}_{\mathrm{T}}\left(\boldsymbol{x}^{\mathscr{A}}\right)-\mathrm{C}_{\mathrm{T}}\left(\boldsymbol{x}^{*}\right)
$$

where $\boldsymbol{x}^{*}$ denotes the optimal solution to (1) in hindsight.

To ease the theoretical analysis, we list a few assumptions on the cost functions $\left\{f_{t}\right\}$ and the feasible set $\mathbb{X}$.

Assumption 1. Cost function $f_{t}(\cdot)$ is $\alpha_{t}$-strongly convex and $l_{t}$-smooth in $\mathbb{R}^{n}$, i.e. for any $x, y \in \mathbb{R}^{n}$, we have $f_{t}(x)+\left\langle\nabla f_{t}(x), y-x\right\rangle+\frac{\alpha_{t}}{2}\|y-x\|^{2} \leq f_{t}(y) \leq f_{t}(x)+\left\langle\nabla f_{t}(x), y-x\right\rangle+\frac{l_{t}}{2}\|y-x\|^{2}$.

In addition, there exist constants $\alpha, l>0$ that do not depend on $T$ such that $\alpha_{t} \geq \alpha$ and $l_{t} \leq l$ for all $1 \leq t \leq T$.

Assumption 2. There exists $G>0$ such that $\left\|\nabla f_{t}(x)\right\| \leq G$ for any $x \in \mathbb{X}$ and any $1 \leq t \leq T$.

Assumption 3. $\mathbb{X}$ is compact with $D:=\max _{x, y \in \mathbb{X}}\|x-y\|$.

We assume Assumption 1 holds throughout the paper. We will explicitly state it when Assumption 2 and 3 are needed.

Finally, we provide two examples for our problem above.

Example 1 (Trajectory Tracking). Consider a dynamical system $x_{t+1}=x_{t}+u_{t}$, where $x_{t}$ is the robot's location, $u_{t}$ is the robot's velocity. Let $y_{t}$ be the target's location. The optimal control problem with tracking error $f_{t}\left(x_{t}\right)=$

\footnotetext{
${ }^{1}$ Predicting the complete function can be challenging, but it simplifies the analysis and it is often practical when the cost functions are parametric [1].

${ }^{2}$ Here we consider $\mathbb{R}^{n}$ because we will use Nesterov's accelerated gradient that requires strong convexity and smoothness outside the feasible set $\mathbb{X}[29]$.
} 
$\frac{1}{2}\left\|x_{t}-y_{t}\right\|^{2}$ and control cost $\frac{\beta}{2}\left\|u_{t}\right\|^{2}=\frac{\beta}{2}\left\|x_{t+1}-x_{t}\right\|^{2}$ can be formulated as

$$
\min _{x_{t} \in \mathbb{X}} \sum_{t=0}^{T-1}\left(f_{t}\left(x_{t}\right)+\frac{\beta}{2}\left\|x_{t+1}-x_{t}\right\|^{2}\right)+f_{T}\left(x_{T}\right),
$$

where $\mathbb{X}$ denotes a feasible set. In reality, a short lookahead window is sometimes available for the target trajectory [30].

Example 2 ( Smoothed regression). Consider a learner who aims to solve a sequence of regression tasks without changing the regressors too much between stages (see e.g. [3], [23]). The problem can be modeled as (1) where $f_{t}(\cdot)$ represents the regression loss function, $x_{t}$ is the regressor at stage $t$, and $\beta$ is the parameter for the smoothing regularization. In some cases, a short lookahead window of future tasks are available, for example, when multiple tasks arrive at the same time but are solved sequentially (see e.g. [31]).

\section{Online Algorithm Design}

This section presents our online algorithms RHGD and RHAG, which are inspired by the offline optimization and offline gradient methods, i.e. gradient descent and Nesterov's accelerated gradient [29] respectively.

\section{A. Offline Optimization and Offline Gradient Descent}

Given all cost functions $f_{1}, \ldots, f_{T}$, the problem (1) becomes a classical optimization problem and can be solved by, e.g., projected gradient descent (GD). The updating rule of GD is the following. For iteration $k=1,2, \ldots$,

$$
\boldsymbol{x}(k)=\Pi_{\mathbb{X} \times \cdots \times \mathbb{X}}\left(\boldsymbol{x}(k-1)-\eta \nabla \mathrm{C}_{\mathbf{T}}(\boldsymbol{x}(k-1))\right),
$$

where $\mathbb{X} \times \cdots \times \mathbb{X}$ denotes the joint feasible set of $\boldsymbol{x}, \eta$ is the stepsize, the initial value $\boldsymbol{x}(0)$ is given. The gradient $\nabla \mathrm{C}_{\mathrm{T}}$ can be evaluated by the partial gradient on each variable $x_{t}$ :

$$
\frac{\partial \mathbf{C}_{\mathbf{T}}}{\partial x_{t}}(\boldsymbol{x})=\nabla f_{t}\left(x_{t}\right)+\beta\left(x_{t}-x_{t-1}\right)+\beta\left(x_{t}-x_{t+1}\right)
$$

when $1 \leq t \leq T-1$, and $\frac{\partial \mathrm{C}_{\mathrm{T}}}{\partial x_{T}}(\boldsymbol{x})=\nabla f_{T}\left(x_{T}\right)+\beta\left(x_{T}-x_{T-1}\right)$ at stage $T$. Notice that the partial gradient $\frac{\partial \mathrm{C}_{\mathrm{T}}}{\partial x_{t}}(\boldsymbol{x})$ only depends on the cost function $f_{t}$ and the stage variables $x_{t-1}, x_{t}, x_{t+1}$. To emphasize this fact, we slightly abuse the notation and write the partial gradient as $\frac{\partial \mathrm{C}_{\mathrm{T}}}{\partial x_{t}}\left(x_{t-1: t+1}\right)$. With this notation, the projected gradient descent (3) can be written equivalently as follows. For iteration $k=1,2, \ldots$, the updating rule of GD on the stage variable $x_{t}$ for $1 \leq t \leq T$ is

$$
x_{t}(k)=\Pi_{\mathbb{X}}\left[x_{t}(k-1)-\eta \frac{\partial \mathrm{C}_{\mathbf{T}}}{\partial x_{t}}\left(x_{t-1: t+1}(k-1)\right)\right]
$$

Rule (4) shows that, to compute $x_{t}(k)$ by the offline gradient descent, we only need $f_{t}$ and $x_{t-1}(k-1), x_{t}(k-$ $1), x_{t+1}(k-1)$, instead of all the cost functions and all the stage variables. This suggests that it is still possible to implement (4) for a few iterations using only the finite lookahead window of cost functions. This is the key insight that motivates our online algorithm design below. 


\section{B. Receding Horizon Gradient Descent (RHGD)}

Inspired by the offline gradient descent, we design our online RHGD (see Algorithm 1). For ease of notation, we define $f_{t}(\cdot):=0$ for $t \leq 0$ or $t>T$ and let $x_{t}(k):=x_{0}$ for $t \leq 0$ and $k \geq 0$ when necessary. At stage $t=1-W$, RHGD sets $x_{1}(0)=x_{0}$. At stage $2-W \leq t \leq T$, RHGD receives $f_{t}, \ldots, f_{t+W-1}$ and runs the following two steps.

In Step 1, RHGD initializes the variable $x_{t+W}(0)$ with an initialization method $\phi$. Notice that $\phi$ can be any method that only uses the available information at $t$, i.e. $f_{1}, \ldots, f_{t+W-1}$ and the stage variables computed before $t$. For instance, $\phi$ can be online gradient descent (OGD), which is a well-known OCO algorithm in literature [5] and is provided below.

$$
x_{t+W}(0)=\Pi_{\mathbb{X}}\left[x_{t+W-1}(0)-\gamma \nabla f_{t+W-1}\left(x_{t+W-1}(0)\right)\right],
$$

where $\gamma>0$ is the stepsize and $x_{t+W-1}(0)$ is available from the Step 1 at the previous stage $t-1$.

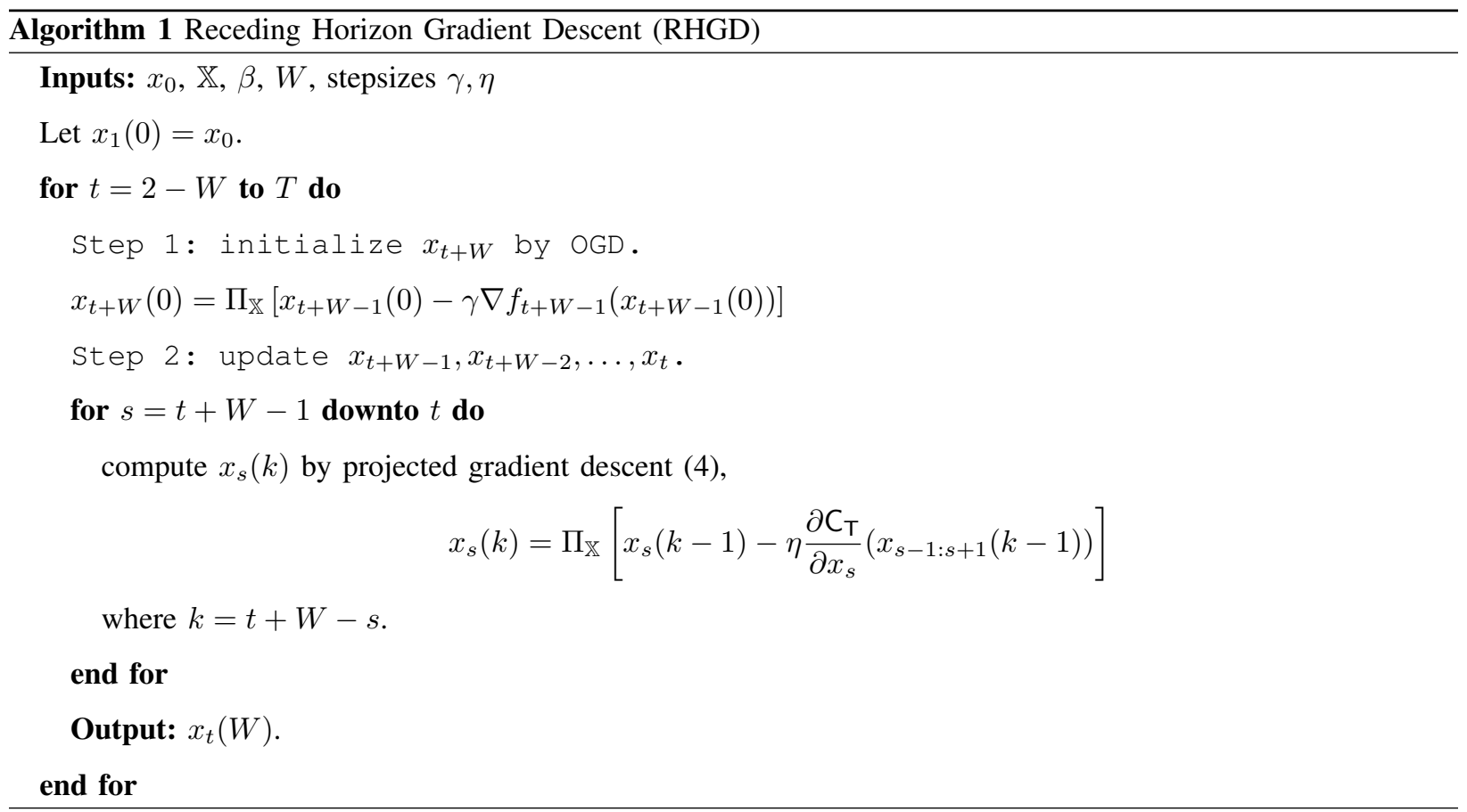

In Step 2, RHGD updates the values of $x_{t+W-1}, x_{t+W-2}, \ldots, x_{t}$ one by one by (4). In the following, we show that Step 2 computes the exact values of $x_{t+W-1}(1), \ldots, x_{t}(W)$ defined in the offline gradient descent (4) for the offine optimization (1) by only using the available online information at $t$. label=-

- At first, RHGD computes $x_{t+W-1}(1)$ exactly by (4) since $f_{t+W-1}$ is received, $x_{t+W}(0)$ is computed in Step $1, x_{t+W-1}(0)$ and $x_{t+W-2}(0)$ have been computed in Step 1 of the stages $t-1$ and $t-2$ respectively.

- Next, RHGD computes $x_{t+W-2}(2)$ exactly by (4) since $f_{t+W-2}$ is received, $x_{t+W-1}(1)$ is computed above, $x_{t+W-2}(1)$ and $x_{t+W-3}(1)$ have been computed in Step 2 of the stages $t-1$ and $t-2$ respectively.

- Similarly, RHGD computes $x_{t+W-3}(3), \ldots, x_{t}(W)$ one by one based on the received costs and the values that have been computed at stages $t, t-1, t-2$. 
The final output at stage $t$ is $x_{t}(W)$, which is the same as that in the $W$ th iteration of the offline gradient descent (4) for $\mathrm{C}_{T}$.

Notice that RHGD (with OGD initialization) only requires $W+1$ projected gradient evaluations at each stage. Therefore, our RHGD is computationally efficient when the projection onto $\mathbb{X}$ can be evaluated efficiently, e.g. when $\mathbb{X}$ is a positive orthant, an n-dimensional box, a probability simplex, a Euclidean ball, etc.

\begin{tabular}{|c|c|c|c|c|c|}
\hline$f_{1}$ & $f_{2}$ & $f_{3}$ & $f_{4}$ & & $\begin{array}{l}t=-1 \\
t=0\end{array}$ \\
\hline$x_{1}(0)$ & $x_{2}(0)$ & $x_{3}(0)$ & $x_{4}(0)$ & $x_{5}(0) t$ & $\begin{array}{l}t=1 \\
t=2\end{array}$ \\
\hline$x_{1}(1)$ & $x_{2}(1)$ & $x_{3}(1)$ & $x_{4}(1)$ & & $t=3$ \\
\hline$x_{1}(2)$ & $x_{2}(2)$ & $x_{3}(2)$ & & & \\
\hline
\end{tabular}

Fig. 1: An example when $W=2$. The new cost function received and the new variables computed by RHGD at stage $t=-1,0,1,2,3$ are marked in different colors.

Example 3 (Illustrative example). Figure 1 provides an illustrative example for RHGD when W=2. Define $x_{t}(k)=x_{0}$ for $t \leq 0, k \geq 0 ; f_{t}=0$ for $t \leq 0$. At $t=-1$, set $x_{1}(0)=x_{0}$.

- At $t=0, R H G D$ receives $f_{0}$ and $f_{1}$. Step 1 initializes $x_{2}(0)$ by OGD (5) with $f_{1}$ and $x_{1}(0)$. Step 2 computes $x_{1}(1)$ by GD (4) with $f_{1}$ and $x_{0}(0), x_{1}(0), x_{2}(0)$; and then computes $x_{0}(2)$ for ease of notation, which is omitted in Fig. 1.

- At $t=1$, RHGD receives $f_{1}, f_{2}$. Step 1 initializes $x_{3}(0)$ by (5) with $f_{2}$ and $x_{2}(0)$. Step 2 first computes $x_{2}(1)$ by (4) with $f_{2}$ and $x_{1}(0), x_{2}(0), x_{3}(0)$ and then computes $x_{1}(2)$ by (4) with $f_{1}$ and $x_{0}(1), x_{1}(1), x_{2}(1) . R H G D$ outputs $x_{1}(2)$.

- Similarly, at $t=2$, RHGD receives $f_{2}, f_{3}$, initializes $x_{4}(0)$, computes $x_{3}(1)$ and then $x_{2}(2)$ by (4), and outputs $x_{2}(2)$.

- Similarly, at $t=3$, RHGD initializes $x_{5}(0)$, computes $x_{4}(1)$ and then $x_{3}(2)$, and outputs $x_{3}(2)$. So on and so forth.

\section{Receding Horizon Accelerated Gradient (RHAG)}

The design idea of RHGD can be extended to other gradient methods, e.g. Nesterov's accelerated gradient (NAG), triple momentum, etc. Due to space limits, we only introduce RHAG (Algorithm 2) based on NAG. NAG updates $x_{t}(k)$ and auxiliary variable $y_{t}(k)$ for $1 \leq t \leq T$ at iteration $k$ by

$$
\begin{aligned}
& x_{t}(k)=\Pi_{\mathbb{X}}\left(y_{t}(k-1)-\eta \frac{\partial \mathrm{C}_{\mathrm{T}}}{\partial x_{t}}\left(y_{t-1: t+1}(k-1)\right)\right), \\
& y_{t}(k)=(1+\lambda) x_{t}(k)-\lambda x_{t}(k-1) .
\end{aligned}
$$

Similar to RHGD, RHAG also conducts two steps at each $t$. The differences are: in Step 1, RHAG initializes not only $x_{t+W}(0)$ but also $y_{t+W}(0)$; and in Step 2, RHAG updates $x_{t+W-1}(1), \ldots, x_{t}(W)$ by NAG (6) instead 
of the gradient descent. Nevertheless, RHAG still outputs $x_{t}(W)$, which is the value of $x_{t}$ after $W$ th iterations of NAG. In total, RHAG also only requires $W+1$ projected gradient evaluations.

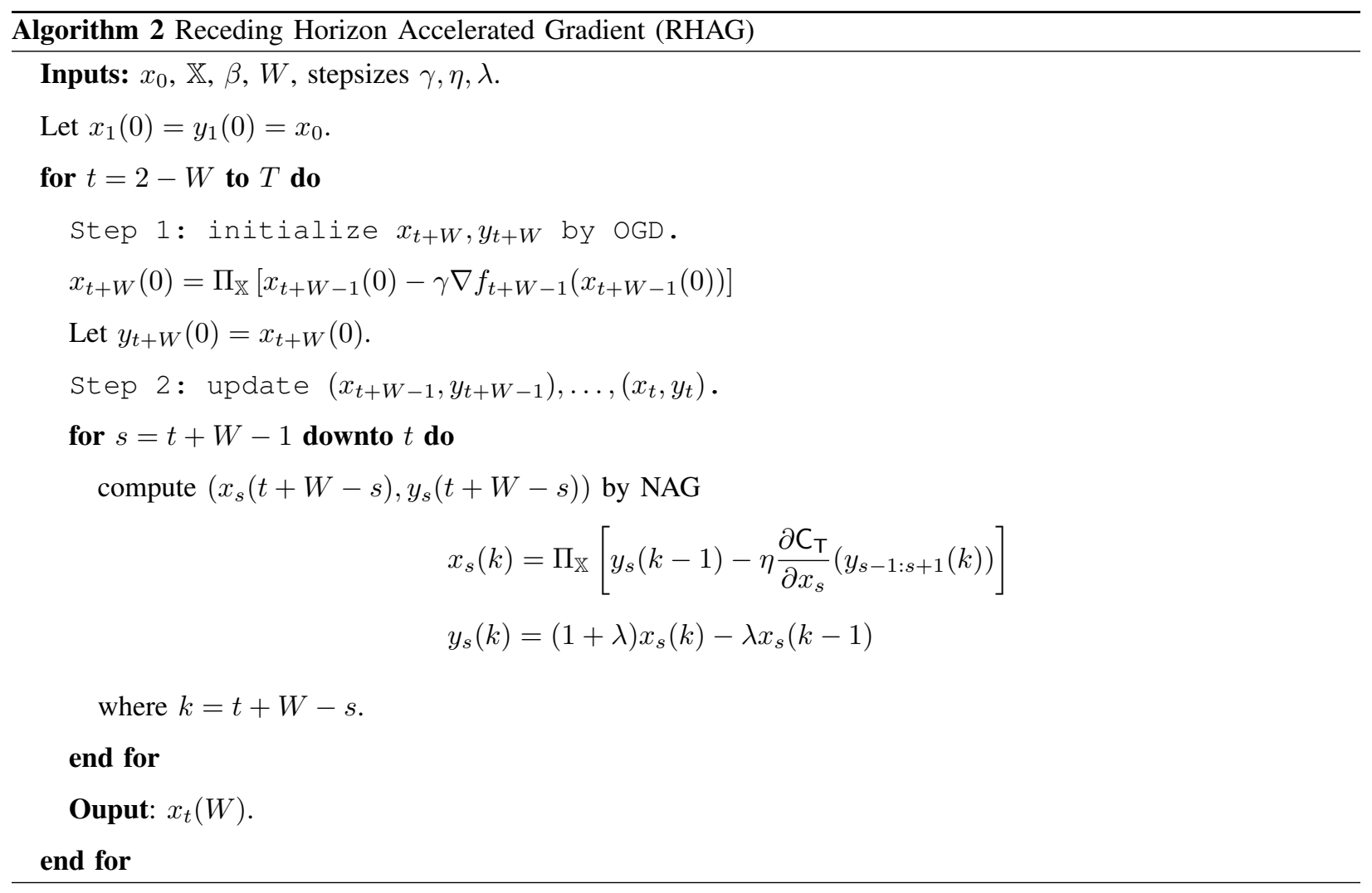

Remark 1. We note that RHGD and RHAG do not use the latest information. To see this, consider Example 3. RHGD updates $x_{3}(1)$ by using $x_{2}(0)$ though $x_{2}(1)$ is available (the same applies to RHAG). This allows RHGD (RHAG) to exactly implement offline $G D(N A G)$ for $\mathrm{C}_{\mathrm{T}}(\boldsymbol{x})$, which greatly simplifies the analysis and maintains the convergence rates of GD (NAG). Numerical results in our online report [32] show that RHGD and its variant that uses the latest information perform similarly, while RHAG outperforms its variant, which is likely due to the sensitivity of NAG to disturbances [33].

\section{Theoretical Analysis of OUR Algorithms}

This section provides the regret upper bounds of RHGD and RHAG, as well as our initialization method OGD. In addition, we provide the stability guarantees of RHGD and RHAG.

We first prove a supportive lemma on the properties of $C_{T}$.

Lemma 1. Given Assumption 1, $\mathrm{C}_{\mathrm{T}}(\boldsymbol{x})$ defined in (1) is $\alpha$-strongly convex and L-smooth on $\mathbb{R}^{n}$ for $L=l+4 \beta$.

Proof. The Hessian of $\sum_{t=1}^{T} \frac{\beta}{2}\left\|x_{t}-x_{t-1}\right\|^{2}$ has eigenvalues in $[0,4 \beta]$ by the Gershgorin circle theorem. Thus, by Assumption $1, \mathrm{C}_{\mathrm{T}}(\boldsymbol{x})$ is $\alpha$ strongly convex and $L=l+4 \beta$ smooth.

Next, we provide the regret upper bounds. 
Theorem 1 (General regret upper bounds). Under Assumption 1 , for $W \geq 0$, given stepsizes $\eta=1 / L$ and $\lambda=\frac{1-\sqrt{\alpha / L}}{1+\sqrt{\alpha / L}}, 3$ for any initialization $\phi$ in Step 1, we have

$$
\begin{aligned}
& \operatorname{Reg}(R H G D) \leq Q_{f}\left(1-\frac{1}{Q_{f}}\right)^{W} \operatorname{Reg}(\phi) \\
& \operatorname{Reg}(R H A G) \leq 2\left(1-\frac{1}{\sqrt{Q_{f}}}\right)^{W} \operatorname{Reg}(\phi)
\end{aligned}
$$

where $Q_{f}=\frac{L}{\alpha}$ and $\operatorname{Reg}(\phi)$ is the regret of implementing the initial values $\left\{x_{t}(0)\right\}_{t=1}^{T}$ computed by the initialization $\phi$.

Proof. Notice that $x_{t}(W)$ is the $W$ th iterate of GD for $\mathrm{C}_{\mathrm{T}}(\boldsymbol{x})$. By GD's convergence rate (see Appendix B in [32]), we have

$$
\begin{aligned}
\operatorname{Reg}(R H G D) & =\mathrm{C}_{\mathbf{T}}(\boldsymbol{x}(W))-\mathrm{C}_{\mathbf{T}}\left(\boldsymbol{x}^{*}\right) \\
& \leq Q_{f}\left(1-1 / Q_{f}\right)^{W}\left[\mathrm{C}_{\mathbf{T}}(\boldsymbol{x}(0))-\mathrm{C}_{\mathbf{T}}\left(\boldsymbol{x}^{*}\right)\right] \\
& =Q_{f}\left(1-1 / Q_{f}\right)^{W} \operatorname{Reg}(\phi)
\end{aligned}
$$

$\operatorname{Reg}(R H A G)$ can be bounded similarly by NAG's convergence rate (see e.g. [29]).

Theorem 2 (Regret upper bounds with OGD initialization). When Assumption 1-3 hold, the regret of the initialization method OGD (5) with stepsize $\gamma=1 / l$ is bounded by, ${ }^{4}$

$$
\operatorname{Reg}(O G D) \leq \delta \sum_{t=1}^{T}\left\|\theta_{t}-\theta_{t-1}\right\|,
$$

where $\delta=(\beta / l+1) \frac{G}{(1-\kappa)}, \kappa=\sqrt{\left(1-\frac{\alpha}{l}\right)}, \theta_{t}=\arg \min _{x \in \mathbb{X}} f_{t}(x)$ for $1 \leq t \leq T$, and $\theta_{0}=x_{0}$.

Consequently, the regrets of RHGD and RHAG with OGD initialization are upper bounded by

$$
\begin{aligned}
& \operatorname{Reg}(R H G D) \leq \delta Q_{f}\left(1-\frac{1}{Q_{f}}\right)^{W} \sum_{t=1}^{T}\left\|\theta_{t}-\theta_{t-1}\right\|, \\
& \operatorname{Reg}(R H A G) \leq 2 \delta\left(1-\frac{1}{\sqrt{Q_{f}}}\right)^{W} \sum_{t=1}^{T}\left\|\theta_{t}-\theta_{t-1}\right\| .
\end{aligned}
$$

The proof of Theorem 2 is deferred to Appendix 1.

Effect of $W$. When $W=0$, RHGD and RHAG reduce to OGD. When $W \geq 0$, RHGD and RHAG's regret bounds exponentially decay with $W$. RHAG's bound decays faster than RHGD's since NAG converges faster than GD.

Path length. The regret bounds in Theorem 2 all linearly depend on $\sum_{t=1}^{T}\left\|\theta_{t}-\theta_{t-1}\right\|$. OGD's bound is similar to the bound for OCO without switching costs [14]. The term $\sum_{t=1}^{T}\left\|\theta_{t}-\theta_{t-1}\right\|$, called the path length in literature [14], consists of (i) $\sum_{t=2}^{T}\left\|\theta_{t}-\theta_{t-1}\right\|$ that captures the variation of the cost functions $\left\{f_{t}\right\}_{t=1}^{T}$, (ii) $\left\|\theta_{1}-\theta_{0}\right\|$ that represents the quality of the initial point $x_{0}$ since $\theta_{0}=x_{0}$. When (i) is close to zero, the path length is dominated by (ii). Otherwise, the path length is dominated by the variation of the cost functions.

\footnotetext{
${ }^{3}$ The stepsize conditions can be relaxed as in [29], yielding different bounds.

${ }^{4}$ Stepsizes $\gamma \leq 1 / l$ also work, yielding different constant factors.
} 
Finally, we provide some stability results of our algorithms.

Corollary 1 (Asymptotic stability). Consider an optimal control problem that is equivalent to problem (1) when $T=\infty$ :

$$
\min _{x_{t} \in \mathbb{X}} \sum_{t=1}^{+\infty}\left[f_{t}\left(x_{t}\right)+\frac{\beta}{2}\left\|u_{t}\right\|^{2}\right] \text {, s.t. } x_{t}=x_{t-1}+u_{t}, \forall t .
$$

Notice that $\theta_{t}:=\arg \min _{\mathbb{X}} f_{t}(x)$ is the optimal steady state for the stage cost at $t$. If $\theta_{t} \rightarrow \theta_{\infty}$ as $t \rightarrow+\infty$ and $\sum_{t=1}^{+\infty}\left\|\theta_{t}-\theta_{t-1}\right\|<+\infty$, then $x_{t}(W) \rightarrow \theta_{\infty}$ as $t \rightarrow+\infty$, where $x_{t}(W)$ denotes the output of RHGD (or RHAG) and $W \geq 0$.

\section{Fundamental Limits}

This section provides fundamental lower bounds on the dynamic regrets for both $W=0$ and $W>0$. For simplicity, we only consider deterministic online algorithms in this paper.

Theorem 3 (No prediction). Consider $W=0$. Given any $T \geq 0, \alpha>0, \beta \geq 0$, a convex compact set $\mathbb{X}$ with diameter $D>0$, and $0 \leq L_{T} \leq D T$, for any deterministic online algorithm $\mathscr{A}$, there exist a sequence of quadratic functions $\left\{f_{t}(\cdot)\right\}_{t=1}^{T}$ with $\alpha$ strong convexity and $\alpha$ smoothness on $\mathbb{R}^{n}$, gradient bound $(3 \alpha+\beta) D$ on $\mathbb{X}$, path length $\sum_{t=1}^{T}\left\|\theta_{t}-\theta_{t-1}\right\| \leq L_{T}$, where $\theta_{t}=\arg \min _{\mathbb{X}} f_{t}(x)$, such that the regret is lower bounded by

$$
\operatorname{Reg}(\mathscr{A}) \geq \zeta D L_{T} \geq \zeta D \sum_{t=1}^{T}\left\|\theta_{t}-\theta_{t-1}\right\|
$$

where $\zeta=\frac{\alpha^{3}(1-\rho)^{2}}{32(\alpha+\beta)^{2}}, \rho=\frac{\sqrt{Q}_{f}-1}{\sqrt{Q}_{f}+1}$, and $Q_{f}=\frac{\alpha+4 \beta}{\alpha}$.

The proof is provided in [32]. Next, we provide some discussion. Notice that $L_{T}$ is an upper bound on the path length $\sum_{t=1}^{T}\left\|\theta_{t}-\theta_{t-1}\right\|$. Theorem 3 shows that the regret lower bound is linear with the path length when $W=0$, which matches OGD's upper bound (9). Besides, Theorem 3 shows that when $L_{T}=\Omega(T)$, no online algorithm can achieve $o(T)$ dynamic regret, which is similar to the claims in [25].

Theorem 4 ( $W$-stage predictions). Given any $T>1,1 \leq W \leq T / 2, \alpha>0, \beta \geq 0$, a convex compact set $\mathbb{X}$ with diameter $D>0,0 \leq L_{T} \leq D T$, for any deterministic online algorithm $\mathscr{A}$, there exist a sequence of quadratic functions $\left\{f_{t}(\cdot)\right\}_{t=1}^{T}$ with $\alpha$ strong convexity and $\alpha$ smoothness on $\mathbb{R}^{n}$, gradient bound $\alpha D$ on $\mathbb{X}$, path length $\sum_{t=1}^{T}\left\|\theta_{t}-\theta_{t-1}\right\| \leq L_{T}$, where $\theta_{t}=\arg \min _{\mathbb{X}} f_{t}(x)$, such that the regret satisfies

$$
\operatorname{Reg}(\mathscr{A}) \geq \begin{cases}\frac{\zeta D}{3} \rho^{2 W} L_{T}, & \text { if } L_{T} \geq D, \\ \frac{\zeta}{3} \rho^{2 W} L_{T}^{2}, & \text { if } L_{T}<D\end{cases}
$$

where $\rho=\frac{\sqrt{Q}_{f}-1}{\sqrt{Q}_{f}+1}, Q_{f}=\frac{\alpha+4 \beta}{\alpha}$, and $\zeta=\frac{\alpha^{3}(1-\rho)^{2}}{32(\alpha+\beta)^{2}}$.

The main proof ideas are provided in Appendix 3.

Special-case analysis. Notice that Theorem 3 and 4 only prove the fundamental lower bounds for the special/worst cases. It is possible to achieve smaller regrets in non-worst cases. 


\begin{tabular}{c|c|c}
\hline Algorithm & $W=5$ & $W=10$ \\
\hline RHGD & $3.48 \times 10^{-4}$ & $6.91 \times 10^{-4}$ \\
RHAG & $5.44 \times 10^{-4}$ & $8.92 \times 10^{-4}$ \\
MPC & $1.67 \times 10^{-1}$ & $3.31 \times 10^{-1}$ \\
\hline
\end{tabular}

TABLE I: Algorithms' running time per stage in seconds.

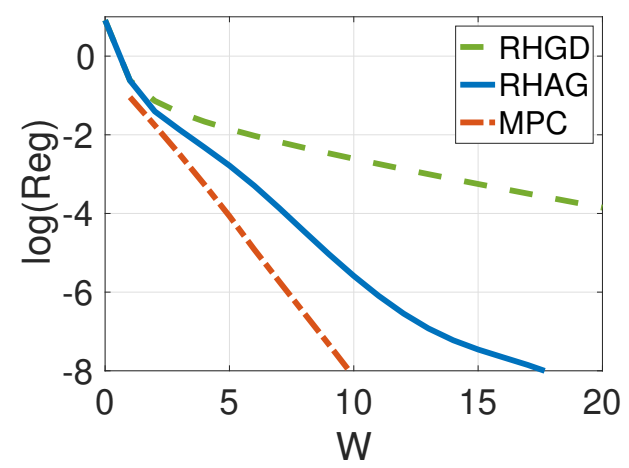

(a) Smoothed regression

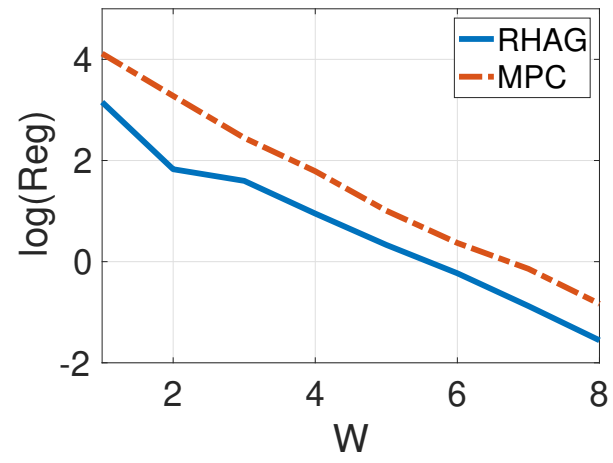

(b) A special example

Fig. 2: The regret of RHGD, RHAG, and MPC with $W$.

Comparion with RHAG. Theorem 4 shows that, given limited predictions $(W \leq T / 2)$, the decay rate of the fundamental lower bound with respect to $W$ is similar to that of RHAG in Theorem 2 for large $Q_{f}$, because to reach the same regret value $R$, the lower bound requires at least $W \geq \Omega\left(\left(\sqrt{Q_{f}}-1\right) \log \left(L_{T} / R\right)\right)$ (by $\rho^{2 W} \geq \exp \left(-\frac{4 W}{\sqrt{Q_{f}}-1}\right)$ ), while RHAG requires at most $W \leq O\left(\sqrt{Q_{f}} \log \left(L_{T} / R\right)\right)$ (by $\left(1-1 / \sqrt{Q_{f}}\right)^{W} \leq \exp \left(-W / \sqrt{Q_{f}}\right)$ ). In addition, given a reasonably large path length $\left(L_{T} \geq D\right)$, the lower bound is linear with the path length, which matches the upper bound of RHAG (11). The comparison above indicates the near-optimality of RHAG at least in the special/worst cases, which may be surprising since RHAG only requires $W+1$ gradient evaluations while online algorithms in Theorem 4 have no computation constraints.

More discussion on $L_{T}$. Interestingly, when $L_{T}<D$, the lower bound in Theorem 4 is quadratic on $L_{T}$ which is smaller than the linear dependence as $L_{T} \rightarrow 0$. This is probably because with a small $L_{T}$, the online problem is "easy" and admits algorithms with better regrets. When $W=0$, the bound is the same for $L_{T}<D$, which is probably because the online algorithms have too limited future information when $W=0$ to benefit from a small $L_{T}$. More discussion is in [32].

\section{NUMERICAL EXPERIMENTS}

This section provides numerical results to complement our theoretical analysis by comparing our algorithms with MPC. 
1) Model predictive control: We consider the following MPC algorithm. At each stage $t$, MPC solves the following open-loop $W$-stage receding horizon optimization

$$
\min _{x_{t} \ldots x_{t+W-1} \in \mathbb{X}} \sum_{\tau=t}^{t+W-1}\left(f_{\tau}\left(x_{\tau}\right)+\frac{\beta}{2}\left\|x_{\tau}-x_{\tau-1}\right\|^{2}\right),
$$

obtains the optimizer $\left\{x_{t}^{t}, \ldots, x_{t+W-1}^{t}\right\}$, and implements $x_{t}^{t}$. Though there are many variants of MPC and several methods to exploit the structures of MPC to speed up its computation, we limit ourselves to solving (15) by the iterative algorithm NAG. Note that at each iteration, NAG requires $W$ gradient evaluations since (15) is a $W$-stage optimization problem. We terminate NAG when the gradient's norm is less than $10^{-14}$.

2) Smoothed regression: Consider Example 2 with a loss function for logistic regression in [34] with an $l_{2}$ regularizer: $f_{t}\left(x_{t}\right)=\frac{1}{M} \sum_{m=1}^{M}\left[\log \left(1+e^{w_{t, m}^{\top} x_{t}}\right)-v_{t, m} w_{t, m}^{\top} x_{t}\right]+\frac{r}{2}\left\|x_{t}\right\|^{2}$, where $M$ is the number of samples, $w_{t, m} \in \mathbb{R}^{n}$ are the features, $v_{t, m} \in\{0,1\}$ are the labels, $r$ is the $l_{2}$ regularization parameter. In particular, we consider $M=60, n=3, T=60, \beta=5$, and $r=0.5$. We generate $\left\{w_{t, m}\right\}_{m=1}^{M}$ as i.i.d. random Gaussian vectors with mean $\mu_{t} \mathbf{1}_{n}$ and covariance $\sigma_{t}^{2} \boldsymbol{I}_{n}$ for each $t$. $\left\{\mu_{t}\right\}_{t=1}^{T}$ are i.i.d. from Unif $[-1,1]$, and $\left\{\sigma_{t}^{2}\right\}_{t=1}^{T}$ are i.i.d. from Unif $[0,1]$. We generate $\left\{v_{t, m}\right\}_{m=1}^{M}$ i.i.d. from $\operatorname{Bern}\left(p_{t}\right)$, where $\left\{p_{t}\right\}_{t=1}^{T}$ are i.i.d. from Unif $[0,1]$. Let $x_{0}=0$, $\mathbb{X}=[-1,1]^{3}$ and choose stepsizes by Theorem 1 and 2 .

Figure 2(a) plots the regrets of RHGD and RHAG with OGD initialization and MPC against $W$. Firstly, the regrets of these algorithms decay linearly on a log scale, indicating the exponential decay with $W$. Further, RHAG decays faster than RHGD, which is consistent with Theorem 2. Besides, there are some fluctuations in RHAG's regret caused by the fluctuations of NAG [35], but RHAG still yields smaller regrets than RHGD. In addition, Figure 2(a) shows that MPC enjoys a better decay rate with $W$. More analysis on MPC's regret is left as future work. Table I compares the running time per stage of RHGD, RHAG, and MPC for $W=5,10$. Notice that RHGD and RHAG are much faster than the MPC described in Section VI.1. This is because our algorithms only evaluate $W+1$ gradients per stage, while we exploit multiple NAG iterations for solving MPC per stage and each iteration requires $W$ gradient evaluations. Nevertheless, we admit that there are other methods and variants of MPC that may further reduce the computation time, which is beyond the scope of this paper.

3) A special example: As indicated in Section V, RHAG is near-optimal among all the deterministic online algorithms at least in the sense of the worst cases. We construct a special case to partially validate this indication by showing that RHAG slightly outperforms MPC in this case. In particular, consider $f_{t}\left(x_{t}\right)=0.5\left(x_{t}-\theta_{t}\right)^{2}$, $\mathbb{X}=[0,6], T=20$. Let $\left\{\theta_{t}\right\}_{t=1}^{T}$ be $[6,0,6,0,6,6,0,6,6,0,6,6,0,6,6,6,6,6,6,6]$. Let $\beta=20$ and $\gamma=0.4$ and choose $\eta$ and $\lambda$ by Theorem 2. Figure 2(b) shows that our gradient-based RHAG achieves slightly better performance than the optimization-based MPC in this case.

\section{CONCLUSION}

This paper attempts to understand the fundamental value of the predictions and to study how to use predictions effectively in the online decision making problems by considering an online convex optimization problem with a $W$-lookahead window on the cost functions and switching costs on the decisions. In this paper, we propose gradient-based online algorithms RHGD and RHAG and provide the dynamic regret upper bounds as well as some 
stability guarantees. We also characterize the fundamental limits of the dynamic regrets of any online algorithms for $W \geq 0$. The fundamental lower bound when $W>0$ is close to RHAG's regret upper bound under certain conditions. There are many interesting future directions, e.g. i) the algorithm design and analysis when there are errors and noises in the implementation, observations, and/or the predictions of the future, in which cases RHGD may be better than RHAG since NAG is sensitive to disturbances; ii) online algorithms when there are coupling constraints across stages and for more general dynamical systems; iii) more analysis on the regrets of MPC and its variants; iv) relaxing the assumptions; v) customizing the lower bounds for different function classes; vi) beyond the worst-case analysis; vii) competitive ratio analysis; viii) algorithm design that involves generating predictions; ix) more discussion on stability.

\section{APPENDIX}

In the following, we provide more discussion for Theorem 3 and Theorem 4 in Appendix A, then provide a convergence rate analysis for projected gradient descent for ease of reference which is used in the proof of Theorem 1. Moreover, we provide a proof for OGD's regret upper bound (9) (see Theorem 2) in Appendix C. In addition, we prove Corollary 1 in Appendix D. Next, we provide a proof of Theorem 4 in Appendix E, where a supportive lemma is proved in Appendix F. Besides, we provide a proof of Theorem 3 in Appendix G. Finally, we provide numerical results as promised in Remark 1 in Appendix H.

\section{A. More discussion on Section V}

We provide more discussion on the case with a small $L_{T}$ in Theorem 3 and Theorem 4 . When $L_{T}$ is small, i.e. $L_{T} \leq D$, the lower bound is $\Omega\left(L_{T}^{2}\right)$ as $L_{T} \rightarrow 0$, which is smaller than $\Omega\left(L_{T}\right)$ because $L_{T}^{2} \leq D L_{T}$. An $O\left(L_{T}^{2}\right)$ upper bound can be achieved by a simple online algorithm: $x_{t}^{\mathscr{A}}=\theta_{t}$. This is verified by the following arguments. Since $\theta_{t}$ minimizes each $f_{t}(\cdot)$, the dynamic regret of $x_{t}^{\mathscr{A}}=\theta_{t}$ is upper bounded by the switching costs, i.e. $\sum_{t=1}^{T} \frac{\beta}{2}\left\|\theta_{t}-\theta_{t-1}\right\|^{2}$, and we have $\sum_{t=1}^{T}\left\|\theta_{t}-\theta_{t-1}\right\|^{2} \leq L_{T}^{2}$. However, when there is no prediction, i.e., $W=0$, this simple online algorithm can not be implemented because $f_{t}(\cdot)$ is not available at stage $t$. In fact, the fundamental limit in Theorem 3 indicates that no online algorithm can achieve $O\left(L_{T}^{2}\right)$ regret as $L_{T} \rightarrow 0$.

\section{B. Convergence rate of projected gradient descent}

The convergence rate analysis for projected gradient descent is standard in literature, but the common guarantee is on the distance between the decision variable and the optimal variable instead of on the gap between the algorithm's cost and the optimal cost (see e.g. [29]). Since the proof of Theorem 1 requires the convergence rate in terms of the function value, for ease of the reader and for the completeness of the paper, we provide a short proof for the convergence rate in terms of the function value below.

Proposition 1 (Convergence rate of projected gradient descent in terms of the function value). Consider constrained optimization $\min _{\mathbb{Q}} f(x)$ where $f(x)$ is $\alpha$ strongly convex and $L$ smooth and $\mathbb{Q}$ is convex. Then, the projected gradient descent on $f(x)$, i.e. $x(k+1)=\Pi_{\mathbb{Q}}\left[x(k)-\frac{1}{L} \nabla f(x(k))\right]$, enjoys the following bound:

$$
f(x(k))-f\left(x^{*}\right) \leq Q_{f}\left(1-1 / Q_{f}\right)^{k}\left[f(x(0))-f\left(x^{*}\right)\right]
$$


where $Q_{f}=1 / L$ and $x^{*}=\arg \min _{\mathbb{Q}} f(x)$.

Proof. Firstly, it can be verified that the projected gradient descent update is equivalent to

$$
x(k)=\underset{x \in \mathbb{Q}}{\arg \min }\left[f\left(x(k-1)+\nabla f(x(k-1))^{\top}(x-x(k-1))+\frac{L}{2}\|x-x(k-1)\|^{2}\right]\right.
$$

Therefore, we have

$$
\begin{aligned}
f(x(k)) & \leq f(x(k-1))+\nabla f(x(k-1))^{\top}(x(k)-x(k-1))+\frac{L}{2}\|x(k)-x(k-1)\|^{2} \\
& =\min _{x \in \mathbb{Q}}\left[f\left(x(k-1)+\nabla f(x(k-1))^{\top}(x-x(k-1))+\frac{L}{2}\|x-x(k-1)\|^{2}\right]\right. \\
& \leq f\left(x(k-1)+\nabla f(x(k-1))^{\top}\left(x^{*}-x(k-1)\right)+\frac{L}{2}\left\|x^{*}-x(k-1)\right\|^{2}\right. \\
& \leq f\left(x^{*}\right)+\frac{L-\mu}{2}\left\|x^{*}-x(k-1)\right\|^{2} \\
& =f\left(x^{*}\right)+\frac{L}{2}\left(1-1 / Q_{f}\right)\left\|x^{*}-x(k-1)\right\|^{2}
\end{aligned}
$$

where the first inequality is by $L$ smoothness and the last inequality is by $\alpha$ strong convexity.

Consequently, we have

$$
\begin{aligned}
f(x(k))-f\left(x^{*}\right) & \leq \frac{L}{2}\left(1-1 / Q_{f}\right)\left\|x^{*}-x(k-1)\right\|^{2} \\
& \leq \frac{L}{2}\left(1-1 / Q_{f}\right)^{k}\left\|x^{*}-x(0)\right\|^{2} \\
& \leq Q_{f}\left(1-1 / Q_{f}\right)^{k}\left(f(x(0))-f\left(x^{*}\right)\right)
\end{aligned}
$$

where the second inequality uses the established convergence rate in Theorem 2.3.4 in [29], i.e.

$$
\left\|x(k)-x^{*}\right\|^{2} \leq\left(1-1 / Q_{f}\right)^{k}\left\|x(0)-x^{*}\right\|^{2}
$$

and the last inequality uses the strong convexity.

\section{Proof of the inequality (9) in Theorem 2}

For simplicity, we slightly abuse the notation and denote OGD's output $\mathbf{x}(0)$ as $\mathbf{x}$ below. We first prove a lemma and then prove (9).

Lemma 2. Given stepsize $\gamma=1 / l$, the outputs of OGD satisfy $\sum_{t=1}^{T}\left\|x_{t}-\theta_{t}\right\| \leq \frac{1}{1-\kappa} \sum_{t=1}^{T}\left\|\theta_{t}-\theta_{t-1}\right\|$ and $\sum_{t=1}^{T}\left\|x_{t}-x_{t-1}\right\|^{2} \leq \frac{2 G}{l(1-\kappa)} \sum_{t=1}^{T}\left\|\theta_{t}-\theta_{t-1}\right\|$

where $x_{1}=\theta_{0}=x_{0}, \kappa=\sqrt{1-\frac{\alpha}{l}}$, and $\theta_{t}=\arg \min _{\mathbb{X}} f_{t}(x)$.

Proof. Firstly, we bound $\sum_{t=1}^{T}\left\|x_{t}-\theta_{t}\right\|$ by the following.

$$
\begin{aligned}
\sum_{t=1}^{T}\left\|x_{t}-\theta_{t}\right\| & \leq \sum_{t=1}^{T}\left\|x_{t}-\theta_{t-1}\right\|+\sum_{t=1}^{T}\left\|\theta_{t-1}-\theta_{t}\right\| \\
& =\sum_{t=2}^{T}\left\|x_{t}-\theta_{t-1}\right\|+\sum_{t=1}^{T}\left\|\theta_{t-1}-\theta_{t}\right\| \\
& \leq \sum_{t=2}^{T} \kappa\left\|x_{t-1}-\theta_{t-1}\right\|+\sum_{t=1}^{T}\left\|\theta_{t-1}-\theta_{t}\right\|
\end{aligned}
$$


where the equality is by $x_{1}=\theta_{0}$, the last inequality is by OGD's updating rule and Theorem 2.2.8 in [29]. ${ }^{5}$ Then, the bound is proved by subtracting $\sum_{t=1}^{T} \kappa\left\|x_{t}-\theta_{t}\right\|$ from the first term and (17), then dividing both sides by $1-\kappa$. Next, we bound $\sum_{t=1}^{T}\left\|x_{t}-x_{t-1}\right\|^{2}$ by the following.

$$
\begin{aligned}
\frac{l}{2} \sum_{t=1}^{T}\left\|x_{t}-x_{t-1}\right\|^{2} & =\frac{l}{2} \sum_{t=2}^{T}\left\|x_{t}-x_{t-1}\right\|^{2}=\frac{l}{2} \sum_{t=1}^{T-1}\left\|x_{t+1}-x_{t}\right\|^{2} \\
& \leq \sum_{t=1}^{T-1}\left[f_{t}\left(x_{t}\right)-f_{t}\left(x_{t+1}\right)\right] \leq \sum_{t=1}^{T-1}\left[f_{t}\left(x_{t}\right)-f_{t}\left(\theta_{t}\right)\right] \\
& \leq \sum_{t=1}^{T-1} G\left\|x_{t}-\theta_{t}\right\| \leq \frac{G}{1-\kappa} \sum_{t=1}^{T}\left\|\theta_{t}-\theta_{t-1}\right\|
\end{aligned}
$$

where the first line uses $x_{1}=x_{0}$, the first inequality uses Corollary 2.2 .1 (2.2.16) in [29], ${ }^{6}$ the second inequality is by $\theta_{t}=\arg \min _{\mathbb{X}} f_{t}(x)$, the third inequality is by $f_{t}\left(x_{t}\right)-f_{t}\left(\theta_{t}\right) \leq\left\langle\nabla f_{t}\left(x_{t}\right), x_{t}-\theta_{t}\right\rangle \leq G\left\|x_{t}-\theta_{t}\right\|$, the last one uses the first bound in Lemma 2.

Now, we can prove (9) in Theorem 2 as follows.

$$
\begin{aligned}
\operatorname{Reg}(O G D) & \leq \sum_{t=1}^{T}\left(f_{t}\left(x_{t}\right)-f_{t}\left(x_{t}^{*}\right)+\frac{\beta}{2}\left\|x_{t}-x_{t-1}\right\|^{2}\right) \\
& \leq \sum_{t=1}^{T}\left(f_{t}\left(x_{t}\right)-f_{t}\left(\theta_{t}\right)+\frac{\beta}{2}\left\|x_{t}-x_{t-1}\right\|^{2}\right) \\
& \leq \sum_{t=1}^{T}\left[G\left\|x_{t}-\theta_{t}\right\|+\frac{\beta}{2}\left\|x_{t}-x_{t-1}\right\|^{2}\right] \\
& \leq \delta \sum_{t=1}^{T}\left\|\theta_{t}-\theta_{t-1}\right\|
\end{aligned}
$$

where the first line uses $\left\|x_{t}^{*}-x_{t-1}^{*}\right\|^{2} \geq 0$, the second line is by $\theta_{t}=\arg \min _{\mathbb{X}} f_{t}(x)$ and thus $f_{t}\left(x_{t}^{*}\right) \geq f_{t}\left(\theta_{t}\right)$, the third inequality is by $f_{t}\left(x_{t}\right)-f_{t}\left(\theta_{t}\right) \leq \nabla f_{t}\left(x_{t}\right)^{\top}\left(x_{t}-\theta_{t}\right) \leq G\left\|x_{t}-\theta_{t}\right\|$, and the last inequality is by Lemma 2.

\section{Proof of Corollary 1}

The equivalence between (12) and (1) is straightforward. $\theta_{t}$ is the optimal steady state at $t$ since

$$
\theta_{t}=\underset{x=x+u \in \mathbb{X}}{\arg \min }\left[f_{t}(x)+\beta / 2\|u\|^{2}\right] .
$$

We denote $\Theta:=\sum_{t=1}^{+\infty}\left\|\theta_{t}-\theta_{t-1}\right\|$ and consider RHGD below. For any $T<\infty$,

$$
\begin{aligned}
\sum_{t=1}^{T-W} \frac{\alpha}{2}\left\|x_{t}(W)-\theta_{t}\right\|^{2} & \leq \sum_{t=1}^{T}\left[f_{t}\left(x_{t}(W)\right)-f_{t}\left(\theta_{t}\right)\right] \\
& \leq \mathrm{C}_{\mathbf{T}}(\mathbf{x}(W))-\mathrm{C}_{\mathbf{T}}(\boldsymbol{\theta})+\frac{\beta}{2} \sum_{t=1}^{T}\left\|\theta_{t}-\theta_{t-1}\right\|^{2} \\
& \leq \operatorname{Reg}(R H G D)+\frac{\beta}{2} \sum_{t=1}^{T}\left\|\theta_{t}-\theta_{t-1}\right\|^{2}
\end{aligned}
$$

${ }^{5}$ Notice that $g_{Q}(\cdot, \cdot)$ in [29] satisfies $g_{Q}\left(x_{t-1}, l\right)=l\left(x_{t-1}-x_{t}\right)$.

${ }^{6}$ This is by $\bar{x}=x_{t}, f=f_{t}, x_{Q}\left(x_{t}, l\right)=x_{t+1}, g_{Q}\left(x_{t}, l\right)=l\left(x_{t}-x_{t+1}\right)$. 


$$
\leq \delta Q_{f}\left[1-\frac{1}{Q_{f}}\right]^{W} \Theta+\frac{\beta}{2} \Theta^{2}
$$

where the first term only considers the summation to $T-W$ because the outputs $x_{t}(W)$ for $t>T-W$ depend on whether RHGD terminates at $T$ or not; the first inequality is by the $\alpha$-strong convexity of $f_{t}(\cdot)$ and the optimality of $\theta_{t}$; the second one is by (1) and $\left\|x_{t}(W)-x_{t-1}(W)\right\|^{2} \geq 0$, and we denote $\boldsymbol{\theta}=\left(\theta_{1}, \ldots, \theta_{T}\right)$; the third inequality is by $\mathbf{C}_{\mathbf{T}}(\boldsymbol{\theta}) \geq \mathbf{C}_{\mathbf{T}}\left(\mathbf{x}^{*}\right)$ and (2); the last line uses Theorem $2, \sum_{t=1}^{T}\left\|\theta_{t}-\theta_{t-1}\right\| \leq \Theta$, and $\sum_{t=1}^{T}\left\|\theta_{t}-\theta_{t-1}\right\|^{2} \leq\left(\sum_{t=1}^{T}\left\|\theta_{t}-\theta_{t-1}\right\|\right)^{2} \leq \Theta^{2}$.

By letting $T \rightarrow+\infty$, we have $\sum_{t=1}^{\infty} \frac{\alpha}{2}\left\|x_{t}(W)-\theta_{t}\right\|^{2} \leq \delta Q_{f}\left(1-1 / Q_{f}\right)^{W} \Theta+\frac{\beta}{2} \Theta^{2}<+\infty$, thus $\left\|x_{t}(W)-\theta_{t}\right\| \rightarrow$ 0 as $t \rightarrow+\infty$. Since $\theta_{t} \rightarrow \theta_{\infty}$, we have $\left\|x_{t}(W)-\theta_{\infty}\right\| \rightarrow 0$. The proof for RHAG is the same.

\section{E. Proof of Theorem 4}

The main proof idea is to construct random cost functions and show that the lower bound (14) holds in expectation, and thus there must exist some case with positive probability such that the lower bound holds.

Without loss of generality, we consider an one dimension case $\mathbb{X}=\left[-\frac{D}{2}, \frac{D}{2}\right]$ and let $x_{0}=0{ }^{7}$

For technical reasons, we consider three cases: (i) when $L_{T} \geq 2 D$, (ii) when $D<L_{T}<2 D$, (iii) when $0 \leq L_{T} \leq D$, and construct slightly different cost function sequences for each case.

Proof for (i) when $L_{T} \geq 2 D$ :

Part 1: construct random $\left\{f_{t}(\cdot)\right\}_{t=1}^{T}$ : For any $\alpha>0, \beta>0$ ( $\beta=0$ is trivial), we construct quadratic function $f_{t}\left(x_{t}\right)=\frac{\alpha}{2}\left(x_{t}-\theta_{t}\right)^{2}$ with parameter $\theta_{t} \in \mathbb{X}$. Thus, $\theta_{t}=\arg \min _{\mathbb{X}} f_{t}\left(x_{t}\right)$. We construct random $\left\{\theta_{t}\right\}_{t=1}^{T}$ as follows. Let $\Delta=\left\lceil T /\left\lfloor L_{T} / D\right\rfloor\right\rceil$ and divide $T$ stages into $K=\left\lceil\frac{T}{\Delta}\right\rceil$ parts, where each part has $\Delta$ stages, except possibly the last part. Notice that $1 \leq K \leq T$ since $1 \leq \Delta \leq T$ when $D \leq L_{T} \leq D T$. For $0 \leq k \leq K-1$, generate $\theta_{k \Delta+1}$ independently by distribution $\mathbb{P}\left(\theta=\frac{D}{2}\right)=\mathbb{P}\left(\theta=-\frac{D}{2}\right)=\frac{1}{2}$, and let $\theta_{t}=\theta_{k \Delta+1}$ for $k \Delta+2 \leq t \leq(k+1) \Delta$.

The next lemma shows that our constructed cost functions satisfy the path length upper bound.

Lemma 3. For $f_{t}\left(x_{t}\right)$ constructed above, the path length of $\left\{f_{t}\right\}_{t=1}^{T}$ satisfies $\sum_{t=1}^{T}\left\|\theta_{t}-\theta_{t-1}\right\| \leq L_{T}$, where $\theta_{0}=x_{0}=0$.

Proof. By the definition of $\Delta$, we have

$$
\Delta=\left\lceil T /\left\lfloor L_{T} / D\right\rfloor\right\rceil \geq T /\left\lfloor L_{T} / D\right\rfloor
$$

Equivalently, we have $\left\lfloor L_{T} / D\right\rfloor \geq T / \Delta$ and thus $\left\lfloor L_{T} / D\right\rfloor \geq\lceil T / \Delta\rceil=K$.

Then, according to the definition of $\theta_{t}$ above, we have

$$
\sum_{t=1}^{T}\left\|\theta_{t}-\theta_{t-1}\right\|=\sum_{k=0}^{K-1}\left\|\theta_{k \Delta+1}-\theta_{k \Delta}\right\| \leq D K \leq D\left\lfloor L_{T} / D\right\rfloor=L_{T}
$$

which completes the proof.

\footnotetext{
${ }^{7}$ Our proof can be extended to any $\mathbb{X} \subseteq \mathbb{R}^{n}$ by letting $f_{t}\left(x_{t}\right)=\alpha / 2\left\|x_{t}-\theta_{t}-\frac{v_{1}+v_{2}}{2}\right\|^{2}$, where $v_{1}, v_{2} \in \mathbb{X}$ and $\left\|v_{1}-v_{2}\right\|=D$, and by generating $\theta_{t}$ randomly with probability $\mathbb{P}\left(\theta=v_{1}\right)=\mathbb{P}\left(\theta=v_{2}\right)=1 / 2$.
} 
Part 2: characterize $\mathbf{x}^{*}$. The problem (1) constructed in Part 1 enjoys a closed-form optimal solution: $\mathbf{x}^{*}=\boldsymbol{\theta} \mathbf{A}^{\top}$, where $\mathbf{x}^{*}=\left(x_{1}^{*}, \ldots, x_{T}^{*}\right)$ denotes the optimal solution as a row vector in $\mathbb{R}^{T} \boldsymbol{\theta}=\left(\theta_{1}, \ldots, \theta_{T}\right), \mathbf{A}=\left(a_{i, j}\right)_{i, j=1}^{T}$. Equivalently, we have $x_{t}^{*}=\sum_{\tau=1-t}^{T-t} a_{t, t+\tau} \theta_{t+\tau}$ so $a_{t, t+\tau}$ represents the influence of $\theta_{t, t+\tau}$ on $x_{t}^{*}$. Lemma 4 will show that the influence $a_{t, t+\tau}$ decays exponentially for $\tau \geq 0$.

Lemma 4. Consider the cost in Part 1. The optimal solution to (1) is $\mathbf{x}^{*}=\boldsymbol{\theta} \mathbf{A}^{\top}$, where $a_{t, t+\tau} \geq \frac{\alpha}{\alpha+\beta}(1-\rho) \rho^{\tau}$ for $\tau \geq 0$.

The proof is provided in Appendix F.

Part 3: characterize $\mathrm{x}^{\mathscr{A}}$. The key observation here is that the output $x_{t}^{\mathscr{A}}$ of any online algorithm $\mathscr{A}$ is a random variable determined by $\left\{\theta_{s}\right\}_{s=1}^{t+W-1}$. This is because $x_{t}^{\mathscr{A}}$ is decided by $\mathscr{A}$ based on $\left\{f_{s}(\cdot)\right\}_{s=1}^{t+W-1}$, which is determined by $\left\{\theta_{s}\right\}_{s=1}^{t+W-1}$ according to the construction in Step $1 .^{8}$

Part 4: lower bound $\mathbb{E}[\operatorname{Reg}(\mathscr{A})]$. To prove the lower bound, we define a set of stages onsider a set of stages

$$
\mathbb{J}:=\{1 \leq t \leq T-W \mid t+W \equiv 1 \quad(\bmod \Delta)\} .
$$

Before the proof of the lower bound, we first prove two helping lemmas.

Lemma 5. Consider the cost in Part 1, for any online algorithm $\mathscr{A}$, we have $\mathbb{E}\left|x_{t}^{\mathscr{A}}-x_{t}^{*}\right|^{2} \geq \frac{a_{t, t+W}^{2} D^{2}}{4}$ for any $t \in \mathbb{J}$, where $a_{t, t+W}$ is an entry of matrix $\mathbf{A}$ defined in Lemma 4.

Proof. We denote the $\sigma$-algebra generated by $\theta_{1}, \ldots, \theta_{t}$ as $\mathscr{F}_{t}$. By our discussion in Part $2, x_{t}^{*}$ is $\mathscr{F}_{T}$-measurable. In addition, by Part 3, for any online algorithm $\mathscr{A}$, the output $x_{t}^{\mathscr{A}}$ is $\mathscr{F}_{t+W-1}$-measurable. It is a classic result that for any $\sigma$-algebra $\mathscr{F}$ of the probability space, the conditional expectation $\mathbb{E}[X \mid \mathscr{F}]$ minimizes the mean square error $E(Y-X)^{2}$ among any random variable $Y$ that is $\mathscr{F}$-measurable (see Theorem 4.1 .15 in [36] for example). Therefore, we have

$$
\mathbb{E}\left(x_{t}^{\mathscr{A}}-x_{t}^{*}\right)^{2} \geq \mathbb{E}\left(\mathbb{E}\left[x_{t}^{*} \mid \mathscr{F}_{t+W-1}\right]-x_{t}^{*}\right)^{2}
$$

Consequently, we do not have to discuss each online algorithm $\mathscr{A}$ but only need to bound $\mathbb{E}\left(\mathbb{E}\left[x_{t}^{*} \mid \mathscr{F}_{t+W-1}\right]-x_{t}^{*}\right)^{2}$, which is provided below.

$$
\begin{aligned}
\mathbb{E}\left(\mathbb{E}\left[x_{t}^{*} \mid \mathscr{F}_{t+W-1}\right]-x_{t}^{*}\right)^{2} & =\mathbb{E}\left(\sum_{\tau=1}^{t+W-1} a_{t, \tau} \theta_{\tau}-\sum_{\tau=1}^{T} a_{t, \tau} \theta_{\tau}\right)^{2}=\mathbb{E}\left(\sum_{\tau=t+W}^{T} a_{t, \tau} \theta_{\tau}\right)^{2} \\
& =\mathbb{E}\left[\left(a_{t, t+W}+\cdots+a_{t, t+W+\Delta-1}\right) \theta_{t+W}\right]^{2}+\mathbb{E}\left(\sum_{\tau=t+W+\Delta}^{T} \theta_{\tau}\right)^{2} \\
& \geq \mathbb{E}\left[a_{t, t+W}^{2} \theta_{t+W}^{2}\right]=a_{t, t+W}^{2} \frac{D^{2}}{4}
\end{aligned}
$$

where the first line is because $x_{t}^{*}=\sum_{\tau=1}^{T} a_{t, \tau} \theta_{\tau}$ as discussed in Part 2 and $\mathbb{E}\left[\theta_{\tau} \mid \mathscr{F}_{t+W-1}\right]=0$ for $\tau \geq t+W$ when $t \in \mathbb{J}$ according to our definition of $\left\{\theta_{t}\right\}_{t=1}^{T}$ in Part 1 ; the second line is because when $t \in \mathbb{J}$, we have

\footnotetext{
${ }^{8}$ Rigorously speaking, to ensure the random variable to be well-defined, some measurability assumption on $\mathscr{A}$ should be imposed, which is satisfied by most algorithms in practice and is thus omitted here for simplicity.
} 
$\theta_{t+W}=\cdots=\theta_{t+W+\Delta-1}$ with mean 0 and $\theta_{t+W}$ is independent of $\theta_{\tau}$ for $\tau \geq t+W+\Delta$ by Part 1 ; the third line is because $a_{t, \tau}>0$ by Lemma 4 , and $\mathbb{E}\left[\theta_{t+W}^{2}\right]=\frac{D^{2}}{4}$ by Part 1 .

Lemma 6. When $T \geq 2 W$ and $L_{T} \geq 2 D$, we have $|\mathbb{J}| \geq \frac{L_{T}}{12 D}$.

Proof. By using the definition of $\mathbb{J}$ and the properties of the floor and ceiling operators, we have

$$
\begin{aligned}
|\mathbb{J}| & =\lceil T / \Delta\rceil-\lceil W / \Delta\rceil \geq\left\lfloor\frac{T-W}{\Delta}\right\rfloor \geq \frac{T-W}{2 \Delta} \\
& \geq \frac{1}{2} \frac{T-W}{T /\left\lfloor L_{T} / D\right\rfloor+1}=\frac{1}{2}\left\lfloor L_{T} / D\right\rfloor \frac{T-W}{T+\left\lfloor L_{T} / D\right\rfloor} \\
& \geq \frac{1}{2}\left\lfloor L_{T} / D\right\rfloor \frac{T-T / 2}{T+T}=\frac{1}{8}\left\lfloor L_{T} / D\right\rfloor \geq \frac{1}{12} L_{T} / D
\end{aligned}
$$

where the first equality can be proved by noticing that

$$
\begin{aligned}
|\mathbb{J}| & =|\{W+1 \leq \tau \leq T \mid \tau \equiv 1(\bmod ) \Delta\}| \\
& =|\{1 \leq \tau \leq T \mid \tau \equiv 1(\bmod ) \Delta\}|-\{1 \leq \tau \leq T \mid \tau \equiv 1(\bmod ) \Delta\} \mid \\
& =\lceil T / \Delta\rceil-\lceil W / \Delta\rceil ;
\end{aligned}
$$

the first inequality is a property of floor and ceiling functons; the second inequality uses the fact that $\lfloor x\rfloor \geq x / 2$ when $x \geq 1$, and that $T-W \geq \Delta$ when $L_{T} \geq 2 D$ and $T \geq 2 W$ because we have $\frac{T}{T-W} \leq 2 \leq\left\lfloor L_{T} / D\right\rfloor$ and thus $T-W=\lceil T-W\rceil \geq\left\lceil T /\left\lfloor L_{T} / D\right\rfloor\right\rceil=\Delta$; the third inequality is by $\Delta=\left\lceil T /\left\lfloor L_{T} / D\right\rfloor\right\rceil \leq T /\left\lfloor L_{T} / D\right\rfloor+1$, the fourth inequality is by $T \geq 2 W$ and $L_{T} \leq D T$, and the last inequality is because $L_{T} / D \geq 2$, and $\lfloor x\rfloor \geq \frac{2}{3} x$ when $x \geq 2$.

Based on the helping lemma above, we can lower bound the regret in expectation.

$$
\begin{aligned}
\mathbb{E}[\operatorname{Reg}(\mathscr{A})] & =\mathbb{E}\left[\mathrm{C}_{\mathrm{T}}\left(\mathbf{x}^{\mathscr{A}}\right)-\mathrm{C}_{\mathrm{T}}\left(\mathbf{x}^{*}\right)\right] \geq \frac{\alpha}{2} \mathbb{E}\left\|\mathbf{x}^{\mathscr{A}}-\mathbf{x}^{*}\right\|^{2} \\
& =\frac{\alpha}{2} \sum_{t=1}^{T} \mathbb{E}\left|x_{t}^{\mathscr{A}}-x_{t}^{*}\right|^{2} \geq \frac{\alpha}{2} \sum_{t \in \mathbb{J}} \mathbb{E}\left|x_{t}^{\mathscr{A}}-x_{t}^{*}\right|^{2} \\
& \geq|\mathbb{J}| \frac{a_{t, t+W}^{2} D^{2} \alpha}{8} \geq \frac{\alpha D}{96}(1-\rho)^{2}\left(\frac{\alpha}{\alpha+\beta}\right)^{2} L_{T} \rho^{2 W}
\end{aligned}
$$

where the first inequality uses Lemma 1, the third one uses Lemma 5, the last one uses Lemma 6 and 4 . Thus, there exists some realization of $\boldsymbol{\theta}$ yielding the lower bound on the regret, which completes the proof when $L_{T} \geq 2 D$. Proof for (ii) when $D<L_{T}<2 D$ : The proof is very similar to the proof above. We also consider cost function $f_{t}\left(x_{t}\right)=\frac{\alpha}{2}\left(x_{t}-\theta_{t}\right)^{2}$, but we define $\left\{\theta_{t}\right\}_{t=1}^{T}$ in a slightly different way, i.e. we let $\theta_{1}=\cdots=\theta_{W}=0$, and $\theta_{W+1}=\cdots=\theta_{T}$ be a random variable following distribution $\mathbb{P}\left(\theta_{t}=\frac{D}{2}\right)=\mathbb{P}\left(\theta_{t}=-\frac{D}{2}\right)=\frac{1}{2}$. It is easy to verify that the upper bound $L_{T}$ on the path length is satisfied: $\sum_{t=1}^{T}\left\|\theta_{t}-\theta_{t-1}\right\|=\left\|\theta_{W+1}\right\|=\frac{D}{2} \leq \frac{L_{T}}{2} \leq L_{T}$. Since the matrix $\mathbf{A}$ does not depend on our choices of $\left\{\theta_{t}\right\}_{t=1}^{T}$, Lemma 4 still holds. In addition, similar to Lemma 5 , we have $\mathbb{E}\left|x_{1}^{\mathscr{A}}-x_{1}^{*}\right|^{2} \geq \frac{a_{1,1+W}^{2} D^{2}}{4}$.

Consequently, we have the lower bound for the regret in expectation by

$$
\mathbb{E}[\operatorname{Reg}(\mathscr{A})]=\mathbb{E}\left[\mathrm{C}_{\mathrm{T}}\left(\mathbf{x}^{\mathscr{A}}\right)-\mathrm{C}_{\mathrm{T}}\left(\mathbf{x}^{*}\right)\right] \geq \frac{\alpha}{2} \mathbb{E}\left|\mathbf{x}^{\mathscr{A}}-\mathbf{x}^{*}\right|^{2} \geq \frac{\alpha}{2} \mathbb{E}\left|x_{1}^{\mathscr{A}}-x_{1}^{*}\right|^{2}
$$




$$
\geq \frac{\alpha a_{1,1+W}^{2} D^{2}}{8} \geq \frac{\alpha D^{2}}{8} \rho^{2 W}(1-\rho)^{2}\left(\frac{\alpha}{\alpha+\beta}\right)^{2} \geq \frac{\alpha D L_{T}}{96} \rho^{2 W}\left(\frac{\alpha(1-\rho)}{\alpha+\beta}\right)^{2}
$$

Proof for (iii) when $0 \leq L_{T} \leq D$ : The proof is very similar to the proof above. We also consider cost function $f_{t}\left(x_{t}\right)=\frac{\alpha}{2}\left(x_{t}-\theta_{t}\right)^{2}$, but we define $\left\{\theta_{t}\right\}_{t=1}^{T}$ in a slightly different way, i.e. we let $\theta_{1}=\cdots=\theta_{W}=0$, and $\theta_{W+1}=\cdots=\theta_{T}$ be a random variable following distribution $\mathbb{P}\left(\theta_{t}=\frac{L_{T}}{2}\right)=\mathbb{P}\left(\theta_{t}=-\frac{L_{T}}{2}\right)=\frac{1}{2}$. It is easy to verify that the upper bound $L_{T}$ on the path length is satisfied: $\sum_{t=1}^{T}\left\|\theta_{t}-\theta_{t-1}\right\|=\left\|\theta_{W+1}\right\|=\frac{L_{T}}{2} \leq L_{T}$. Since the matrix $\mathbf{A}$ does not depend on our choices of $\left\{\theta_{t}\right\}_{t=1}^{T}$, Lemma 4 still holds. In addition, similar to Lemma 5 , we have $\mathbb{E}\left|x_{1}^{\mathscr{A}}-x_{1}^{*}\right|^{2} \geq \frac{a_{1,1+W}^{2} L_{T}^{2}}{4}$.

Consequently, we have the lower bound for the regret in expectation by

$$
\begin{aligned}
\mathbb{E}[\operatorname{Reg}(\mathscr{A})] & =\mathbb{E}\left[\mathrm{C}_{\mathrm{T}}\left(\mathbf{x}^{\mathscr{A}}\right)-\mathrm{C}_{\mathrm{T}}\left(\mathbf{x}^{*}\right)\right] \geq \frac{\alpha}{2} \mathbb{E}\left|\mathbf{x}^{\mathscr{A}}-\mathbf{x}^{*}\right|^{2} \geq \frac{\alpha}{2} \mathbb{E}\left|x_{1}^{\mathscr{A}}-x_{1}^{*}\right|^{2} \\
& \geq \frac{\alpha a_{1,1+W}^{2} L_{T}^{2}}{8} \geq \frac{\alpha L_{T}^{2}}{8} \rho^{2 W}(1-\rho)^{2}\left(\frac{\alpha}{\alpha+\beta}\right)^{2} \geq \frac{\alpha L_{T}^{2}}{96} \rho^{2 W}\left(\frac{\alpha(1-\rho)}{\alpha+\beta}\right)^{2}
\end{aligned}
$$

\section{F. Proof of Lemma 4}

The proof takes four steps:

(I) study unconstrained optimization and show that $\tilde{\boldsymbol{x}}^{*}=\arg \min _{\mathbb{R}^{T}} \mathrm{C}_{\mathrm{T}}(\boldsymbol{x})=\boldsymbol{\theta} \boldsymbol{A}^{\top}$.

(II) show that the constrained optimization admits the same optimal solution: $\boldsymbol{x}^{*}=\tilde{\boldsymbol{x}}^{*}$

(III) give closed-form expression for matrix $\boldsymbol{A}$

(IV) lower bound the entries $a_{t, t+\tau}$ for $\tau \geq 0$ of matrix $\boldsymbol{A}$

(I) Unconstrained optimization $\arg \min _{\mathbb{R}^{T}} \mathrm{C}_{\mathrm{T}}(x)=A \theta$.

By (1) and Part 1 in Appendix E, we have that

$$
\mathrm{C}_{\mathbf{T}}(\boldsymbol{x})=\sum_{t=1}^{T}\left[f_{t}\left(x_{t}\right)+\frac{\beta}{2}\left\|x_{t}-x_{t-1}\right\|^{2}\right]=\sum_{t=1}^{T}\left[\frac{\alpha}{2}\left\|x_{t}-\theta_{t}\right\|^{2}+\frac{\beta}{2}\left\|x_{t}-x_{t-1}\right\|^{2}\right] .
$$

Since $C_{T}(\boldsymbol{x})$ is strongly convex, the optimal solution to $\min _{\mathbb{R}^{T}} \mathrm{C}_{\mathrm{T}}(\boldsymbol{x})$ can be defined by the first-order condition below:

$$
\begin{aligned}
& \alpha\left(\tilde{x}_{t}-\theta_{t}\right)+\beta\left(2 \tilde{x}_{t}-\tilde{x}_{t-1}-\tilde{x}_{t+1}\right)=0, \quad t=1, \ldots, T-1 \\
& \alpha\left(\tilde{x}_{T}-\theta_{T}\right)+\beta\left(\tilde{x}_{T}-\tilde{x}_{T-1}\right)=0
\end{aligned}
$$

By $x_{0}=\theta_{0}=0$ and canceling $\alpha$ on both sides, we can write the linear equation systems in the matrix form: $\tilde{\boldsymbol{x}}^{*} \boldsymbol{H}^{\top}=\boldsymbol{\theta}$, where $\boldsymbol{x}, \boldsymbol{\theta}$ are row vectors, $\boldsymbol{H}$ is given as below:

$$
\boldsymbol{H}=\left(\begin{array}{ccccc}
1+2 \frac{\beta}{\alpha} & -\frac{\beta}{\alpha} & 0 & \cdots & 0 \\
-\frac{\beta}{\alpha} & 1+2 \frac{\beta}{\alpha} & -\frac{\beta}{\alpha} & \cdots & 0 \\
0 & -\frac{\beta}{\alpha} & 1+2 \frac{\beta}{\alpha} & \cdots & 0 \\
\vdots & \vdots & \vdots & \ddots & \vdots \\
0 & 0 & 0 & \cdots & 1+\frac{\beta}{\alpha}
\end{array}\right)
$$


Notice that $\boldsymbol{H}$ is strictly diagonally dominant, so $\boldsymbol{H}$ is invertible. Therefore, the optimal solution to the unconstrained optimization, $\tilde{\boldsymbol{x}}^{*}=\arg \min _{\mathbb{R}^{T}} \mathrm{C}_{\mathrm{T}}(\boldsymbol{x})$, is given by

$$
\tilde{\boldsymbol{x}}^{*}=\boldsymbol{\theta} \boldsymbol{A}^{\top} \quad \text { where } \boldsymbol{A}:=\boldsymbol{H}^{-1}
$$

(II) The constrained optimization has the same solution. Since $\boldsymbol{H}$ is strictly diagonally dominant, then by Theorem 1 in [37], we have

$$
\|\boldsymbol{A}\|_{\infty}=\left\|\boldsymbol{H}^{-1}\right\|_{\infty} \leq \max _{1 \leq t \leq T} \frac{1}{\left|h_{t t}\right|-\sum_{s \neq t}\left|h_{t, s}\right|}=1
$$

Besides, since $\boldsymbol{H}$ has negative off-diagonal entries and positive diagonal entries, and is strictly diagonally dominant, the inverse of $\boldsymbol{H}$, denoted by $\boldsymbol{A}$ now, is nonnegative. Therefore, for each $t, \tilde{x}_{t}^{*}$ can be written as a convex combination of elements in $\mathbb{X}$ :

$$
\tilde{x}_{t}^{*}=\sum_{s=1}^{T} a_{t, s} \theta_{s}+\left(1-\sum_{s=1}^{T} a_{t, s}\right) 0
$$

because $\theta_{t} \in \mathbb{X}$ and $0 \in \mathbb{X}$. By the convexity of $\mathbb{X}$, we have $\tilde{x}_{t}^{*} \in \mathbb{X}$, then naturally, $\tilde{x}^{*} \in \mathbb{X} \times \cdots \times \mathbb{X}$. As a result, $\boldsymbol{x}^{*}=\arg \min _{\mathbb{X} \times \cdots \times \mathbb{X}} \mathrm{C}_{\mathrm{T}}(\boldsymbol{x})=\arg \min _{\mathbb{R}^{T}} \mathrm{C}_{\mathrm{T}}(\boldsymbol{x})=\tilde{\boldsymbol{x}}^{*}=\boldsymbol{\theta} \boldsymbol{A}^{\top}$.

\section{(III) Closed form expression of $A$.}

Since matrix $\boldsymbol{H}$ has many good properties, such as strictly diagonal dominance, positive diagonally entries and negative off-diagonal entries, tridiagonality, symmetry, we can find a closed-form expression for its inverse, denoted by $\boldsymbol{A}$ now, according to Theorem 2 in [38]. In particular, the entries of $A$ are given by $a_{t, t+\tau}=\frac{\alpha}{\beta} w_{t} v_{t+\tau}$ for $\tau \geq 0$ where

$$
\begin{aligned}
& w_{t}=\frac{\rho}{1-\rho^{2}}\left(\frac{1}{\rho^{t}}-\rho^{t}\right) \quad v_{T}=\frac{1}{-w_{T-1}+(\xi-1) w_{t}} \\
& v_{t}=c_{3} \frac{1}{\rho^{T-t}}+c_{4} \rho^{T-t} \quad c_{3}=v_{T}\left(\frac{(\xi-1) \rho-\rho^{2}}{1-\rho^{2}}\right) \quad c_{4}=v_{T} \frac{1-(\xi-1) \rho}{1-\rho^{2}}
\end{aligned}
$$

and $\rho=\frac{\sqrt{Q_{f}}-1}{\sqrt{Q_{f}}+1}, \xi=\alpha / \beta+2$. Since $A$ is nonnegative and $w_{t}$ is apparently positive, we have $v_{t} \geq 0$ for all $t$.

(IV) Lower bound $a_{t, t+\tau}$ for $\tau \geq 0$.

We will bound $w_{t}, v_{T}$ and $v_{t+\tau} / v_{T}$ separately and then combine them together for a lower bound of $a_{t, t+\tau}$ for $\tau \geq 0$.

First, we bound $w_{t}$ by

$$
\rho^{t} w_{t}=\frac{\rho}{1-\rho^{2}}\left(1-\rho^{2 t}\right) \geq \rho
$$

since $t \geq 1$ and $\rho<1$.

Next, we bound $v_{T}$ in the following way:

$$
\begin{aligned}
\rho^{-T} v_{T} & =\frac{1}{(\xi-1)\left(1-\rho^{2 T}\right)-\left(\rho-\rho^{2 T-1}\right)} \frac{1-\rho^{2}}{\rho} \\
& \geq \frac{1}{(\xi-1)\left(1-\rho^{2 T}\right)} \frac{1-\rho^{2}}{\rho} \geq \frac{1}{\xi-1} \frac{1-\rho^{2}}{\rho}
\end{aligned}
$$

where $\xi=\frac{\alpha}{\beta}+2=\frac{2 Q_{f}+2}{Q_{f}-1}, \rho=\frac{\sqrt{Q_{f}}-1}{\sqrt{Q_{f}}+1}$; the first inequality is by $T \geq 1,\left(\rho-\rho^{2 T-1}\right) \geq 0$; the second inequality is by $0<\rho<1$. 
Then, we bound $v_{t+\tau} / v_{T}$.

$$
\begin{aligned}
\rho^{T-t-\tau} \frac{v_{t+\tau}}{v_{T}} & =\left(\frac{(\xi-1) \rho-\rho^{2}}{1-\rho^{2}}\right)+\frac{1-(\xi-1) \rho}{1-\rho^{2}} \rho^{2(T-t-\tau)} \\
& \geq\left(\frac{(\xi-1) \rho-\rho^{2}}{1-\rho^{2}}\right)=\left(\frac{\rho^{2}+1-\rho-\rho^{2}}{1-\rho^{2}}\right)=\frac{1-\rho}{1-\rho^{2}}
\end{aligned}
$$

where the inequality is by $1-(\xi-1) \rho \geq 0, v_{T} \geq 0$, and the second equality is by $\rho^{2}-\xi \rho+1=0$.

Finally, combining three parts together,

$$
\begin{aligned}
a_{t, t+\tau} & =\frac{\alpha}{\beta}\left[\rho^{t} w_{t}\right]\left[\rho^{-T} v_{T}\right]\left[\rho^{T-t-\tau} \frac{v_{t+\tau}}{v_{T}}\right] \rho^{\tau} \\
& \geq \frac{\alpha}{\beta} \rho \frac{1}{\xi-1} \frac{1-\rho^{2}}{\rho}\left(\frac{1-\rho}{1-\rho^{2}}\right) \rho^{\tau}=\frac{\alpha}{\alpha+\beta}(1-\rho) \rho^{\tau}
\end{aligned}
$$

\section{G. Proof of Theorem 3}

The proof is similar to the proof of Theorem 4. We will also construct random cost functions and prove the lower bound of the regret in expectation. We will discuss two scenarios: $0<L_{T}<D$, and $D \leq L_{T} \leq D T$ ( $L_{T}=0$ is trivially true), and construct different function sequences to prove the lower bound. Without loss of generality, we let $x_{0}=0$.

Scenario 1: $0<L_{T}<D$.

Construction of random costs. For each $0<L_{T}<D$, we consider the following construction of $\mathbb{X} \subseteq \mathbb{R}^{2}$ :

$$
\mathbb{X}=\left[-\frac{L_{T}}{2}, \frac{L_{T}}{2}\right] \times\left[-\frac{\sqrt{D^{2}-L_{T}^{2}}}{2}, \frac{\sqrt{D^{2}-L_{T}^{2}}}{2}\right]
$$

It is easy to verify that the diameter of $\mathbb{X}$ is $D$.

For any $\alpha>0$, consider the parametrized cost function:

$$
f_{t}\left(x_{t}, y_{t} ; \tilde{x}_{t}, \tilde{y}_{t}\right)=\frac{\alpha}{2}\left(x_{t}-\tilde{x}_{t}\right)^{2}+\frac{\alpha}{2}\left(y_{t}-\tilde{y}_{t}\right)^{2}
$$

where $\tilde{x}_{t} \in[-M, M]$ for $M=D+(1+\beta / \alpha) \frac{L_{T}}{2}$ and $\tilde{y}_{t} \in\left[-\frac{D}{2}, \frac{D}{2}\right]$. It is easy to verify that the gradient bound is $G=\alpha \sqrt{(M+D / 2)^{2}+D^{2}} \leq(3 \alpha+\beta) D$.

Next, we consider two possible function sequences, and each sequence happens with probability 1/2.

Sequence 1: $\tilde{x}_{1}=M, \tilde{x}_{t}=\frac{L_{T}}{2}$ for $t \geq 2 . \tilde{y}_{t}=0, t \in[T]$.

Sequence 2: $\tilde{x}_{1}=-M, \tilde{x}_{t}=-\frac{L_{T}}{2}$ for $t \geq 2 . \tilde{y}_{t}=0, t \in[T]$.

Let $\left(\theta_{t}, \varphi_{t}\right)=\arg \min _{\mathbb{X}} f_{t}\left(x_{t}, y_{t} ; \tilde{x}_{t}, \tilde{y}_{t}\right)$, and $\left(\boldsymbol{x}^{*}, \boldsymbol{y}^{*}\right):=\left(x_{1}, y_{1}, \ldots, x_{T}, y_{T}\right)=\arg \min _{\mathbb{X} \times \ldots \mathbb{X}} \mathbf{C}_{\mathbf{T}}(\boldsymbol{x}, \boldsymbol{y})$. Then, for each sequence of the cost functions, the optimal solutions $\left(\boldsymbol{x}^{*}, \boldsymbol{y}^{*}\right)$ are

Sequence 1: $\theta_{t}=x_{t}^{*}=\frac{L_{T}}{2}, \varphi_{t}=y_{t}^{*}=0$ for $1 \leq t \leq T$.

Sequence 2: $\theta_{t}=x_{t}^{*}=-\frac{L_{T}}{2}, \varphi_{t}=y_{t}^{*}=0$ for $1 \leq t \leq T$.

Bound $\mathbb{E}\left[C_{\mathrm{T}}\left(\boldsymbol{x}^{\mathscr{A}}, \boldsymbol{y}^{\mathscr{A}}\right)-\mathrm{C}_{\mathrm{T}}\left(\boldsymbol{x}^{*}, \boldsymbol{y}^{*}\right)\right]$.

By strong convexity, we have

$$
\mathbb{E}\left[\mathrm{C}_{\mathrm{T}}\left(\boldsymbol{x}^{\mathscr{A}}, \boldsymbol{y}^{\mathscr{A}}\right)-\mathrm{C}_{\mathrm{T}}\left(\boldsymbol{x}^{*}, \boldsymbol{y}^{*}\right)\right] \geq \mathbb{E} \sum_{t=1}^{T}\left[\frac{\partial \mathrm{C}_{\mathrm{T}}}{\partial x_{t}}\left(\boldsymbol{x}^{*}, \boldsymbol{y}^{*}\right)\left(x_{t}^{\mathscr{A}}-x_{t}^{*}\right)+\frac{\partial \mathrm{C}_{\mathrm{T}}}{\partial y_{t}}\left(x^{*}, y^{*}\right)\left(y_{t}^{\mathscr{A}}-y_{t}^{*}\right)\right]
$$




$$
\begin{aligned}
& \geq \mathbb{E}\left[\frac{\partial \mathrm{C}_{\mathrm{T}}}{\partial x_{1}}\left(x^{*}, y^{*}\right)\left(x_{1}^{\mathscr{A}}-x_{1}^{*}\right)\right] \\
& =\frac{1}{2}(-h)\left(x_{1}^{\mathscr{A}}-\frac{L_{T}}{2}\right)+\frac{1}{2} h\left(x_{1}^{\mathscr{A}}+\frac{L_{T}}{2}\right)=\frac{1}{2} h L_{T} \\
& \geq \frac{\alpha D}{2} L_{T} \geq \frac{\alpha D L_{T}}{32}(1-\rho)^{2}\left(\frac{\alpha}{\alpha+\beta}\right)^{2}
\end{aligned}
$$

where the second inequality is by $\frac{\partial \mathrm{C}_{\mathrm{T}}}{\partial y_{t}}\left(\boldsymbol{x}^{*}, \boldsymbol{y}^{*}\right)=0$ when $t \geq 1$, and $\frac{\partial \mathrm{C}_{\mathrm{T}}}{\partial x_{t}}\left(\boldsymbol{x}^{*}, \boldsymbol{y}^{*}\right)\left(x_{t}^{\mathscr{A}}-x_{t}^{*}\right)=0$ when $t \geq 2$; in the first equality, $h=\frac{\partial \mathrm{C}_{\mathrm{T}}}{\partial x_{1}}\left(\boldsymbol{x}^{*}, \boldsymbol{y}^{*}\right)$ when the costs follow Sequence 2, so $\frac{\partial \mathrm{C}_{\mathrm{T}}}{\partial x_{1}}\left(\boldsymbol{x}^{*}, \boldsymbol{y}^{*}\right)=-h$ when the costs follow Sequence 1; the third inequality is by $h \geq \alpha D$; the last inequality is to be consistent with the bound in Scenario 2 .

Scenario 2: $D \leq L_{T} \leq D T$. The proof is the same as the proof of Theorem 4 except for one difference: when $W=0$, we are able to give a better bound for $|J|$ even without the condition $L_{T} \geq 2 D$.

The bound for $|J|$ is given below.

Lemma 7. When $T \geq 1$, and $D \leq L_{T} \leq D T$, we have $|\mathbb{J}| \geq \frac{L_{T}}{4 D}$.

Proof. By definition of $\mathbb{J}$ and $\Delta=\left\lceil T /\left\lfloor L_{T} / D\right\rfloor\right\rceil \leq T /\left\lfloor L_{T} / D\right\rfloor+1$, when $L_{T} \geq D$ and $T \geq 1$, we have

$$
\begin{aligned}
|\mathbb{J}| & =\left\lceil\frac{T}{\Delta}\right\rceil \geq \frac{T}{\Delta} \geq \frac{T}{T /\left\lfloor L_{T} / D\right\rfloor+1} \\
& =\left\lfloor L_{T} / D\right\rfloor \frac{T}{T+\left\lfloor L_{T} / D\right\rfloor} \geq \frac{L_{T}}{2 D} \frac{T}{T+T}=\frac{L_{T}}{4 D}
\end{aligned}
$$

by $\lfloor x\rfloor \geq x / 2$ when $x \geq 1$, and $L_{T} \leq D T$.

Then, the lower bound on the expected regret can be proved in the same way as in the proof of Theorem 4.

$$
\mathbb{E}\left[C_{\mathbf{T}}\left(\boldsymbol{x}^{\mathscr{A}}\right)-\mathrm{C}_{\mathbf{T}}\left(\boldsymbol{x}^{*}\right)\right] \geq \mathbb{E} \frac{\alpha}{2}\left\|\boldsymbol{x}^{\mathscr{A}}-\boldsymbol{x}^{*}\right\|^{2} \geq \frac{\alpha}{2} \sum_{t \in \mathbb{J}} \frac{a_{t, t}^{2} D^{2}}{4} \geq \frac{\alpha D L_{T}}{32}(1-\rho)^{2}\left(\frac{\alpha}{\alpha+\beta}\right)^{2}
$$

\section{H. Additional numerical results for the last paragraph of Section III}

In the last paragraph of Section III, we mention that our RHGD and RHAG do not use the latest information, i.e. RHGD computes $x_{s}(k)$ with $x_{s-1}(k-1)$ in Step 2 even though $x_{s-1}(k)$ is available (RHAG is similar). In this section, we provide numerical results that compare RHGD and RHAG with their variants that use the latest information, i.e. computing $x_{s}(k)$ with $x_{s-1}(k)$ in Step 2 of RHGD (RHAG is similar). The setup of our numerical experiment is the same as Section VI.2.

In Figure 3, we compare RHGD, RHAG with their variants that use the most recent information, i.e. RHGDrecent and RHAG-recent. It can be observed that by utilizing the most recent information, the performance of RHGD-recent and RHGD are similar. However, RHAG-recent performs much worse than RHAG. Some intuitive explanation is provided below. Since RHAG implements the offline Nesterov's accelerated gradient (NAG) exactly, RHAG-recent can be viewed as the offline NAG with some errors/disturbances in the inputs at each iteration. It is well-known that NAG is sensitive to disturbances and small disturbances can worsen the performance a lot [33]. This might explain why RHAG-recent performs much worse than RHAG. 


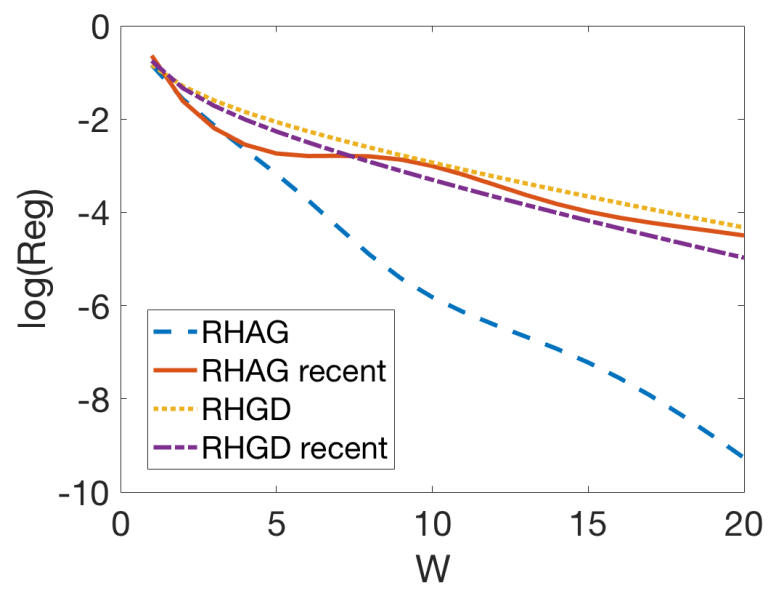

Fig. 3: Comparison between RHGD, RHAG and their variants that use the most recent information.

\section{REFERENCES}

[1] M. Lin, Z. Liu, A. Wierman, and L. L. Andrew, "Online algorithms for geographical load balancing," in 2012 international green computing conference (IGCC). IEEE, 2012, pp. 1-10.

[2] M. Tanaka, "Real-time pricing with ramping costs: A new approach to managing a steep change in electricity demand," Energy Policy, vol. 34, no. 18, pp. 3634-3643, 2006.

[3] F. Zenke, B. Poole, and S. Ganguli, "Continual learning through synaptic intelligence," in Proceedings of the 34th International Conference on Machine Learning-Volume 70, 2017, pp. 3987-3995.

[4] J. Rios-Torres and A. A. Malikopoulos, "A survey on the coordination of connected and automated vehicles at intersections and merging at highway on-ramps," IEEE Transactions on Intelligent Transportation Systems, vol. 18, no. 5, pp. 1066-1077, 2016.

[5] E. Hazan, Introduction to Online Convex Optimization. Now Publishers, 2016.

[6] N. Chen, J. Comden, Z. Liu, A. Gandhi, and A. Wierman, "Using predictions in online optimization: Looking forward with an eye on the past," in Proceedings of the 2016 ACM SIGMETRICS International Conference on Measurement and Modeling of Computer Science. ACM, 2016, pp. 193-206.

[7] J. Rawlings and D. Mayne, "Postface to model predictive control: Theory and design," Nob Hill Pub, pp. 155-158, 2012.

[8] A. Alessio and A. Bemporad, "A survey on explicit model predictive control," in Nonlinear model predictive control. Springer, 2009, pp. 345-369.

[9] M. Kögel and R. Findeisen, "Stabilization of inexact MPC schemes," in 53rd IEEE Conference on Decision and Control. IEEE, 2014, pp. 5922-5928.

[10] Y. Wang and S. Boyd, "Fast model predictive control using online optimization," IEEE Transactions on Control Systems Technology, vol. 18 , no. 2, pp. 267-278, 2010.

[11] K. Graichen and A. Kugi, "Stability and incremental improvement of suboptimal MPC without terminal constraints," IEEE Transactions on Automatic Control, vol. 55, no. 11, pp. 2576-2580, 2010.

[12] S. Paternain, M. Morari, and A. Ribeiro, "A prediction-correction method for model predictive control," in 2018 Annual American Control Conference (ACC). IEEE, 2018, pp. 4189-4194.

[13] M. Diehl, R. Findeisen, F. Allgöwer, H. G. Bock, and J. P. Schlöder, "Nominal stability of real-time iteration scheme for nonlinear model predictive control," IEEE Proceedings-Control Theory and Applications, vol. 152, no. 3, pp. 296-308, 2005.

[14] A. Mokhtari, S. Shahrampour, A. Jadbabaie, and A. Ribeiro, "Online optimization in dynamic environments: Improved regret rates for strongly convex problems," in 55th IEEE Conference on Decision and Control (CDC). IEEE, 2016, pp. 7195-7201.

[15] M. Ellis and P. D. Christofides, "Economic model predictive control with time-varying objective function for nonlinear process systems," AIChE Journal, vol. 60, no. 2, pp. 507-519, 2014. 
[16] A. Ferramosca, D. Limon, and E. F. Camacho, "Economic MPC for a changing economic criterion for linear systems," IEEE Transactions on Automatic Control, vol. 59, no. 10, pp. 2657-2667, 2014.

[17] D. Angeli, A. Casavola, and F. Tedesco, "Theoretical advances on economic model predictive control with time-varying costs," Annual Reviews in Control, vol. 41, pp. 218-224, 2016.

[18] A. Alessandretti, A. P. Aguiar, and C. N. Jones, "On convergence and performance certification of a continuous-time economic model predictive control scheme with time-varying performance index," Automatica, vol. 68, pp. 305-313, 2016.

[19] L. Grüne and S. Pirkelmann, "Economic model predictive control for time-varying system: Performance and stability results," Optimal Control Applications and Methods, 2018.

[20] V. M. Zavala and M. Anitescu, "Real-time nonlinear optimization as a generalized equation," SIAM Journal on Control and Optimization, vol. 48, no. 8, pp. 5444-5467, 2010.

[21] M. Zanon, S. Gros, and M. Diehl, "A Lyapunov function for periodic economic optimizing model predictive control," in 52nd IEEE Conference on Decision and control. IEEE, 2013, pp. 5107-5112.

[22] Y. Li, X. Chen, and N. Li, "Online optimal control with linear dynamics and predictions: Algorithms and regret analysis," in Advances in Neural Information Processing Systems, 2019, pp. 14858-14870.

[23] G. Goel and A. Wierman, "An online algorithm for smoothed regression and LQR control," Proceedings of Machine Learning Research, vol. 89, pp. 2504-2513, 2019.

[24] A. Rakhlin and K. Sridharan, "Online learning with predictable sequences," in Conference on Learning Theory, 2013, pp. 993-1019.

[25] O. Besbes, Y. Gur, and A. Zeevi, "Non-stationary stochastic optimization," Operations research, vol. 63, no. 5, pp. 1227-1244, 2015.

[26] L. Andrew, S. Barman, K. Ligett, M. Lin, A. Meyerson, A. Roytman, and A. Wierman, "A tale of two metrics: Simultaneous bounds on competitiveness and regret," in Conference on Learning Theory, 2013, pp. 741-763.

[27] A. Simonetto, A. Mokhtari, A. Koppel, G. Leus, and A. Ribeiro, "A class of prediction-correction methods for time-varying convex optimization," IEEE Transactions on Signal Processing, vol. 64, no. 17, pp. 4576-4591, 2016.

[28] Y. Tang, E. Dall'Anese, A. Bernstein, and S. Low, "Running primal-dual gradient method for time-varying nonconvex problems," arXiv preprint arXiv:1812.00613, 2018.

[29] Y. Nesterov, Introductory lectures on convex optimization: A basic course. Springer Science \& Business Media, 2013, vol. 87.

[30] R. Rosales and S. Sclaroff, "Improved tracking of multiple humans with trajectory prediction and occlusion modeling," Boston University Computer Science Department, Tech. Rep., 1998.

[31] A. Pentina, V. Sharmanska, and C. H. Lampert, "Curriculum learning of multiple tasks," in Proceedings of the IEEE Conference on Computer Vision and Pattern Recognition, 2015, pp. 5492-5500.

[32] Y. Li, G. Qu, and N. Li, "Online optimization with predictions and switching costs: Fast algorithms and the fundamental limit," arXiv preprint arXiv:1801.07780, 2018.

[33] O. Devolder, F. Glineur, and Y. Nesterov, "First-order methods of smooth convex optimization with inexact oracle," Mathematical Programming, vol. 146, no. 1-2, pp. 37-75, 2014.

[34] (2012) Logistic regression. [Online]. Available: http://www.stat.cmu.edu/ $\sim \sim$ cshalizi/uADA/12/lectures/ch12.pdf

[35] W. Su, S. Boyd, and E. Candes, "A differential equation for modeling nesterov's accelerated gradient method: Theory and insights," in Advances in Neural Information Processing Systems, 2014, pp. 2510-2518.

[36] R. Durrett, Probability: theory and examples. Cambridge university press, 2019, vol. 49.

[37] J. M. Varah, "A lower bound for the smallest singular value of a matrix," Linear Algebra and its Applications, vol. 11, no. 1, pp. 3-5, 1975.

[38] P. Concus, G. H. Golub, and G. Meurant, "Block preconditioning for the conjugate gradient method," SIAM Journal on Scientific and Statistical Computing, vol. 6, no. 1, pp. 220-252, 1985. 